\title{
Mean Field Analysis of Low-Dimensional Systems
}

\author{
L. Chayes \\ Department of Mathematics, UCLA, Los Angeles, CA 90059-1555, USA. \\ E-mail: 1chayes@math.ucla.edu
}

Received: 15 August 2007 / Accepted: 15 April 2009

Published online: 21 August 2009 - (C) The Author(s) 2009. This article is published with open access at Springerlink.com

\begin{abstract}
For low-dimensional systems, (i.e. 2D and, to a certain extent, 1D) it is proved that mean-field theory can provide an asymptotic guideline to the phase structure of actual systems. In particular, for attractive pair interactions that are sufficiently "spead out" according to an exponential (Yukawa) potential it is shown that the energy, free energy and, in particular, the block magnetization (as defined on scales that are large compared with the lattice spacing but small compared to the range of the interaction) will only take on values near to those predicted by the associated mean-field theory. While this applies for systems in all dimensions, the significant applications are for $d=2$ where it is shown: (a) If the mean-field theory has a discontinuous phase transition featuring the breaking of a discrete symmetry then this sort of transition will occur in the actual system. Prominent examples include the two-dimensional $q=3$ state Potts model. (b) If the mean-field theory has a discontinuous transition accompanied by the breaking of a continuous symmetry, the thermodynamic discontinuity is preserved even if the symmetry breaking is forbidden in the actual system. E.g. the two-dimensional $O(3)$ nematic liquid crystal. Further it is demonstrated that mean-field behavior in the vicinity of the magnetic transition for layered Ising and XY systems also occurs in actual layered systems (with spread-out interactions) even if genuine magnetic ordering is precluded.
\end{abstract}

\section{Introduction}

Mean-field theory has traditionally proved to be a reliable guide for predicting, on a coarse level, the behavior in realistic systems. In particular, the location and order of a phase transition may be confidently - if not always accurately - ascertained for a given system by performing the associated mean-field calculation. In recent years some mathematical underpinnings for these trends have been provided in [2,3] (with some ideas therein dating back to [21]). Specifically, the tendency for discontinuous transitions in "realistic" systems was, to a certain extent, elucidated by a comparison to mean-field 
theory. The results of [2] may be summarized, roughly, as follows: If $\mathcal{H}$ is the Hamiltonian for an nearest neighbor attractive (ferromagnetic) spin-system on $\mathbb{Z}^{d}$ and the associated mean-field theory has a discontinuous transition at temperature $T_{\mathrm{MF}}$ then, for large dimensions, the actual model has a discontinuous transition at (a normalized) temperature $T_{d} \rightarrow T_{\mathrm{MF}}$ as $d \rightarrow \infty$. Further, as $d \rightarrow \infty$, the observable characteristics of the system, e.g. latent heat, response functions, etc. approach the corresponding characteristics predicted by the mean-field theory. The nearest neighbor assumption in [2] was to ensure the condition of reflection positivity; $d \geq 3$ was required for the convergence of certain $\left(k\right.$-space) integrals and, further, $d^{-1} \ll 1$ was used as a small parameter. In [3], the large $d$ condition was relaxed by the consideration of exponentially decaying interactions but the condition $d \geq 3$ was still required.

In this note, the obvious step of combining reflection positivity techniques with contour methods will be taken. Thus, at least for models with (exponentially) spread-out interactions, this allows the extensions of the results in [2 and 3] to $d=2-$ and, in a weak sense, even to $d=1$. In particular, at the foundation of this note, is the result that at length-scales that are "large" - but still small compared with the range of the interaction - the average value of the order parameter is asymptotically close to that predicted by mean-field theory. Foremost, this will be used to demonstrate that in a variety of $2 \mathrm{D}$ models with discontinuous transition in their associated mean-field theories, there is an actual discontinuous transition provided that the range parameter of the interaction is sufficiently large. It is remarked that this statement includes $2 \mathrm{D}$ models where the breaking of a continuous symmetry, usually associated with an ordered low temperature phase, is necessarily absent. Of course if a discrete symmetry is broken, a magnetized phase is supported in $d=2$ and several of the better known transitions of this sort will be discussed as well. As a pertinent example it is established (but not for the first time, cf. the discussion below) that a $2 \mathrm{D}$, three-state Potts model has a first order transition.

As for one dimension, since the range of the interaction is ultimately finite, there will be no transitions of any sort. Still, the results concerning the various observables and the associated thermodynamic potentials - apply. Explicitly, in the vicinity of $T_{\mathrm{MF}}$, there is some sort of pseudo-transition even though all thermodynamic quantities are analytic. While this result is of certain modest æsthetic appeal, it is also pertinent to the study of layered systems.

An idealized layered system is a $d$-dimensional system that is extended $\mathrm{L}$ units in the $d+1^{\text {st }}$ direction; the physically relevant cases concern $d=1$ and $d=2$. While systems of this sort were an important showcase for scaling theory and the renormalization group (see e.g. [24] and, especially, [27] and references therein) it seems that till [10], an honest mean-field theory for systems of this sort had never been derived. In the reference [10], (see also [17]) an issue of seminal importance concerned the transition temperature in the layer. For certain systems, e.g. Ising and XY, (where the spins are unit vectors in $n=1$ and $n=2$ dimensions respectively and the ordinary dot product is used to define the interaction) the transition temperature was found to deviate from the $d+1$-dimensional bulk temperature by an amount $t$ given by

$$
|t| \sim \mathrm{C} \frac{\pi^{2}}{\mathrm{~L}^{2}},
$$

where the constant $\mathrm{c}$ is system specific, but explicitly computable, and the asymptotic symbol pertains to the limit $L \gg 1$. The result in Eq. (1.1) was required to understand the thinning of ${ }^{4} \mathrm{He}$ layers in the vicinity of the bulk superfluid transition temperature as observed in the cold-temperature experiments of Garcia, Chen and co-workers $[15,16]$. 
It would seem that the implicit assumption (or philosophy) behind the analysis is that at the shifted temperature, the layered system actually undergoes a magnetic-type transition. This is false in two-dimensions for XY systems and patently false for any one-dimensional system. However, the overall magnetic - or superfluid - properties of the layer are not, as it turns out, quite so relevant as the free energy per particle within the layer as compared to that of the bulk. For layered systems, by and large, the script follows that which was described above: For a sufficiently spread-out interaction, there is indeed a local ordering that is governed by mean-field theory and the free energetics - which is imminently associated with this local ordering - is accurately described by the layered mean-field theory. Thus, it can be claimed, the mean-field understanding of the layer thinning has some mathematical justification in the context of more realistic systems.

It should be emphasized that there is - among others - one severe limitation to this work: The entire approach is contour based and the relevant estimates are enacted via reflection positivity. Hence, to the author's knowledge, this limits us to attractive pair interactions with only one mechanism for spreading out the interaction, namely using the Yukawa ${ }^{1}$ (exponential) coupling. (Precise definitions follow in the next section.) By effective contrast: Recently - concurrent with the writing of the present work - a proof of $1^{\text {st }}$ order transitions for some $q \geq 3$-state Potts models in $d \geq 2$ has been announced [18]. In this approach, which bears certain similarities to the present one, contour estimates are performed with Pirogov-Sinai based methods. Thus, while the work in [18] is ostensibly limited to a single model and, also, a single method to spreadout the interaction, the technique is inherently more flexible. Indeed it seems, albeit with tremendous labor, that these methods might be adapted to a wider variety of interactions and be used to analyze any number of mean-field-type phase transitions associated with the breakdown of discrete symmetries.

Let us close this section first, with an informal survey of various results that will be established and then an organizational outline. To start off: for models of a particular type with range parameter $\mu^{-1} \gg 1$, it will be shown that on large blocks the spatially averaged magnetization must, with high probability, be close to a value predicted by the associated mean-field theory. Energy, free energy and other thermodynamic quantities follow suit and the result holds in all dimensions. Thus, if the mean-field theory has a transition at some $T_{\mathrm{MF}}$ there is evidently some sort of transition-like behavior in the spread-out system even if all thermodynamic quantities are analytic as in $d=1$ or known to be smooth and with no actual magnetic transition like the standard $O(n)$ spin-systems in $d=2$.

In $d \geq 2$ the above considerations allow the proof of first order magnetic transitions in models with discrete symmetries, in particular the Potts models for $q \geq 3$ and the cubic models for $r \geq 4$. For systems such as the $O(n)$ nematic models with continuous symmetries and first order transitions (for $n \geq 2$ ) in the mean-field theory, the thermodynamic component of the transition, if nothing else, will persist in $d \geq 2$, especially $d=2$. These transitions will be accompanied by a discontinuity in the local magnetization notwithstanding that other considerations may rule out the possibility of a global magnetization. Models without any particular symmetry can also be treated and a particular example of a tertiary alloy will be discussed.

\footnotetext{
1 In addition, one can augment or replace the exponential interaction with interactions that decay as a power of the distance; this was a mechanism employed in [3] for treating lower dimensional systems. However, in the unreformed opinion of the author, power law potentials effectively change the dimension of the system and, in any case, cannot be construed as a finite range interactions.
} 
Finally, layered Ising and XY systems will be treated. A mean-field transition temperature, related to the minimal eigenvalue of a certain 1D Laplacian has been calculated in [10] for these systems. By standard methods, it will be shown that this is an upper bound on the actual critical temperature and in the Ising case, for large $\mu^{-1}$, it is asymptotically a lower bound. More importantly, for both systems it will be proved that the free energy of the actual system is close to that predicted by mean-field theory.

The remainder of this paper will be organized as follows: In Sect. 2, first the finitedimensional ("realistic") models under consideration will be defined in generality along with some necessary formalism and working notation. Then there will be a subsect. 2.2, devoted to mean-field theory. This will start with some concise definitions and then, within the context of the theory, a definition of (scenario for) a generic $1^{\text {st }}$ order transition. In Subsect. 2.3, layered systems will be described in some limited generality sufficient to discuss the results derived in [10]. At this point, enough notation will have been established so that by Sect. 3, we are ready for precise statements of theorems. In Sect. 3, all theorems stated will all be of a general nature. The main result will be that if a mean-field model has a generic $1^{\text {st }}$ order scenario then the corresponding "realistic" system will also have this transition $d \geq 2$ provided that the range parameter is sufficiently large. A series of propositions and corollaries then follow which cover, in general terms, all items in the above summary. Sect. 4 is devoted to statements about the specific systems mentioned above. Sect. 5 is for proofs. Subsects. 5.1 and 5.2 will be devoted to statements that concern magnetics and energetics respectively; the latter can be omitted without too much loss of continuity. In Subsect. 5.3 proofs of the main general results will be provided and, in Subsect. 5.4 all results concerning specific systems will be established. Finally, Subsect. 5.5 will consist of a brief appendix devoted to some elementary properties of the mean-field theory formalism.

\section{Definitions and Setup}

Here we will fix notation, define briefly a working version of mean-field theory and provide an abbreviated description of layered systems.

2.1. Background. The basic setup will be pretty much the same as in [2] (and [3]). In particular, we will be discussing spin-systems where the spin variables reside in a compact $\Omega$, which is a subset of a finite-dimensional vector space $\mathbb{E}_{\Omega}$ that is endowed with a positive definite inner product $(-\cdot-)$. Spin variables, generically denoted by an $\mathbf{s}$, are distributed according to some a priori measure denoted by $\alpha_{0}(-)$. The formal Hamiltonian on $\mathbb{Z}^{d}$ is given by

$$
-\mathcal{H}=\sum_{i, j} J_{i, j}\left(\mathbf{s}_{i} \cdot \mathbf{s}_{j}\right),
$$

where each pair of sites is counted once and the $J_{i, j} \geq 0$. It may, on occasion, be desirable to add an external field to the interaction. Thus, if $b \in \mathbb{E}_{\Omega}$ we may add to $-\beta \mathcal{H}$ the term $\sum_{i}\left(b \cdot \mathbf{s}_{i}\right)$. However unless the external field represents a parameter of the model that we wish to actively vary, the field term will be implicitly incorporated into the single-spin measure. This work will be exclusively concerned with the so called Yukawa interactions for which the $J_{i, j}$ are given by

$$
J_{i, j}=K(\mu) e^{-\mu|i-j|_{1}} .
$$


Here $\mu>0$ is the Yukawa parameter - to be considered small - and unless otherwise specified, $K(\mu)$ is chosen so that $\sum_{i \neq j} J_{i, j}=1$. Finite volume Gibbs states and their infinite-volume limits are defined in the standard fashion. Here, for reasons that may already be clear, there will be a vested interest in toroidal measures. In this context, with interactions ranging well beyond nearest neighbor, the convention that will be used, for any given rectangular $\Lambda \subset \mathbb{Z}^{d}$, is to periodically repeat the spin configuration and count all the interactions between spins in $\Lambda$ and the image spins in $\Lambda^{c}$ as dictated by the contents of Eqs. (2.1)-(2.2). The Gibbs measures for such "finite volume" spin configurations $\mathbf{s}_{\Lambda}$ at inverse temperature $\beta$ using the toroidal extension $\mathbb{T}_{\Lambda}$ will be denoted by $\alpha_{\beta, \mathbb{T}_{\Lambda}} ;$ that is to say

$$
\alpha_{\beta, \mathbb{T}_{\Lambda}}\left(\mathbf{s}_{\Lambda}\right) \propto e^{-\beta \mathcal{H}_{\Lambda}\left(\mathbf{s}_{\Lambda}\right)},
$$

where $\mathcal{H}_{\Lambda}\left(\mathbf{s}_{\Lambda}\right)$ denotes the extended periodic interaction as described above. For most purposes, tori with all linear dimensions the same will be sufficient and the corresponding measures, for tori of scale $L$, will be denoted by $\alpha_{\beta, \mathbb{T}_{L}}$. The normalization constant for the weights in Eq. (2.3) - the partition function - will be denoted by $Z_{\Lambda, \beta}$ or $Z_{L, \beta}$ as appropriate. For additional notational continuity, see [2] Sect. 1.2.

2.2. Mean-field theory. Mean-field theory for a Hamiltonian of the form Eq. (2.1) is defined as follows: If $\beta$ denotes the usual temperature parameter and $m \in \operatorname{Conv}(\Omega)$, the free energy function is defined to be $-\frac{1}{2} \beta(m, m)-S(m)$ which are, respectively, the energy and entropy terms. The latter will be discussed momentarily, the former will be denoted by $-\frac{1}{2} \beta m^{2}$. This combination of energy and entropy will be denoted by $\Phi_{\beta}(m)$, the actual mean-field free energy is defined by minimizing $\Phi_{\beta}(m)$ :

$$
F_{\mathrm{MF}}(\beta)=\inf _{m \in \operatorname{Conv}(\Omega)} \Phi_{\beta}(m)=\inf _{m \in \operatorname{Conv}(\Omega)}-\left[\frac{1}{2} \beta m^{2}+S(m)\right] .
$$

The entropy is defined, intrinsically, by

$$
S(m)=\inf _{h \in \mathbb{E}_{\Omega}}[G(h)-(m, h)],
$$

where $e^{G(h)}=\int_{\Omega} \alpha_{0}(d s) e^{(s \cdot h)}$.

The entropy is concave which makes the overall combination of $\Phi_{\beta}(m)$ an interesting playoff between a convex and concave piece. We denote by $\mathcal{M}_{\beta}$ the set of minimizing magnetizations. It is not difficult to see that $\mathcal{M}_{\beta}$ is non-empty and, obviously, confined to the set

$$
\mathscr{C}_{\Omega}=\{m \in \operatorname{Conv}(\Omega) \mid S(m)>-\infty\},
$$

where the entropy is finite. Various convexity/continuity properties will be discussed in a brief appendix; for all intents and purposes we may restrict attention to the interior of $\mathscr{C}_{\Omega}$ on which $\Phi_{m}(\beta)$ is a continuous function.

In mean-field theory, first order transitions come about due to an exchange of minima. The structure of mean-field theory is analytically simple enough so that for first order transitions, the following scenario would seem to be generic:

Definition 2.1. Generic MF first order scenario: 
(i) At some value of the temperature parameter, $\beta_{t}^{\mathrm{MF}}$, a degeneracy in the minima of $\Phi_{\beta_{t}^{\mathrm{MF}}}(m)$ has occurred. Namely there are two non-empty sets, $M_{I}$ and $M_{I I}$ in $\operatorname{Conv}(\Omega)$ that are separated such that $\mathcal{M}_{\beta_{t}^{\mathrm{MF}}}=M_{I} \cup M_{I I}$. (These sets need not themselves be connected.)

(ii) For all $\zeta>0$ sufficiently small, there is an interval $\left[\beta_{I}, \beta_{I I}\right]$ with $\beta_{t}^{\mathrm{MF}} \in\left(\beta_{I}, \beta_{I I}\right)$, and separated sets $\mathbb{M}_{I}$ and $\mathbb{M}_{I I}$ such that

(a) $M_{I} \subset \mathbb{M}_{I}$ and $M_{I I} \subset \mathbb{M}_{I I}$.

(b) At $\beta=\beta_{I}, \mathbb{M}_{I}$ contains all the minimizers of $\Phi_{\beta}(m)$ and similarly for $\mathbb{M}_{I I}$ at $\beta=\beta_{I I}$. In particular, at $\beta=\beta_{I}, \Phi_{\beta_{I}}(m)>F_{\mathrm{MF}}\left(\beta_{I}\right)+\zeta$ for all $m \in \mathbb{M}_{I I}$ and similarly, for $\mathbb{M}_{I}$ at $\beta=\beta_{I I}$.

(c) Each $m \in \mathbb{M}_{I} \cup \mathbb{M}_{I I}$ if and only if for some $\beta \in\left[\beta_{I}, \beta_{I I}\right] \Phi_{\beta}(m)-F_{\mathrm{MF}}(\beta)<$ $\zeta$.

(d) Adopting, temporarily, notation for the $\zeta$ dependence of the items described in (a) and (b), then, as $\zeta \downarrow 0$, we have $\mathbb{M}_{I}^{\zeta} \rightarrow M_{I}$ (in the sense $\cap_{\zeta>0} \mathbb{M}_{I}^{\zeta}=M_{I}$ ) and $\mathbb{M}_{I I} \rightarrow M_{I I}$ while $\left[\beta_{I}^{\zeta}, \beta_{I I}^{\zeta}\right] \rightarrow\left\{\beta_{t}^{\mathrm{MF}}\right\}$.

Remark 1. It does not seem possible, armed with only the unadorned definitions of this section, to prove a general theorem to the effect that all mean-field first order transitions follow the generic scenario. On the other hand, it is difficult to imagine a mean-field theory of the above type describing a first order transition that is not of this kind. Indeed, as we shall see in the proofs for specific systems, very little is used about the actual systems beyond the occurrence of the first order transition itself. Notwithstanding, some small knowledge of $\mathcal{M}_{\beta}$ in the vicinity of $\beta_{t}$ is inevitably required and therefore one is forced into a case-by-case analysis. Fortunately, much of the difficult work along these lines has already been performed in [2 and 3].

Remark 2. It is further remarked that in the above definition, the temperature parameter has been chosen as the driving parameter for the simple reason that temperature driven transitions are more dramatic and hence better known. In mean-field theory, a first order transition can occur with the variation of other coupling parameters and, with obvious adjustments of notation, a first order scenario can be defined accordingly. Indeed, later on, there will be occasion to use the field driven version of the above first order scenario.

2.3. Layered systems. As mentioned earlier, a general mean-field approach for layered systems has been initiated in [10]. Of course (as discussed in [10]) such systems have been analyzed in the physics literature. But ultimately these analyses rely on independent notions of scaling - all of which turn out to be true. However, as an upshot, they lack in quantitative predictive power (e.g. the coefficient in the shift of the transition temperature for critical layered systems). It should be mentioned that the work in [10] pertains to the analysis of a particular experimental set-up and thus, as far as generalities are concerned, is only of a preliminary nature. Hence, for present purposes, we will be content to discuss an abbreviated version of some ultimate "general theory" for layered systems. In particular, it will be ensured by fiat that the formulation of layered systems fits immediately into the existing framework. The results herein will be sufficient to vindicate the calculations contained in [10] and, it should be mentioned, this was the initial motivation for the current work.

The starting point is $L$ copies of $\mathbb{Z}^{d}$ which should be regarded, in a natural fashion, as a subset of $\mathbb{Z}^{d+1}$. For physical applications, one would usually take $d$ equal one or 


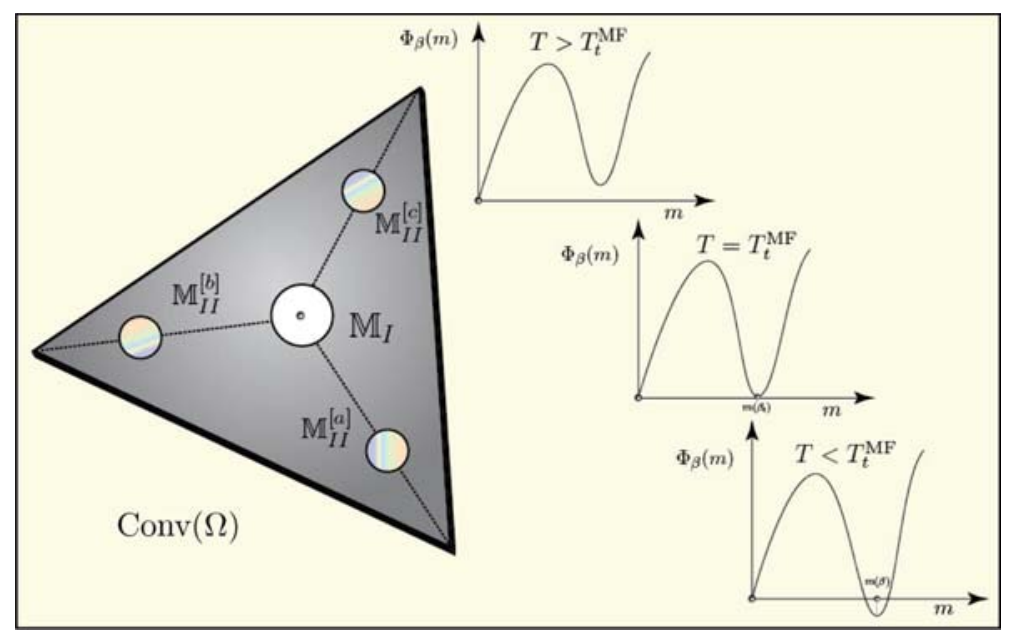

Fig. 1. First order scenario for the 3-state Potts model: The space $\Omega$ may be taken as the vertices of an equilateral triangle. For $\beta<\beta_{t}^{\mathrm{MF}}=4 \log 2$ the unique global minimizer is $m=0$ which at $\beta_{t}^{\mathrm{MF}}$ becomes degenerate with three secondary minima located a distance of $\frac{5}{9}$ along the axes of the triangle. These three points represent the set $M_{I I}$, the set $M_{I}$ is simply the origin. Insert shows the mean-field free energy as a function of the scalar magnetization concentrated along one of these axes (going off-axis only increases the free energy). The generic first order scenario follows easily from analytic considerations of [2]. Theorem 4.1 establishes a first order transition in the $2 \mathrm{D}$ version of this model with Yukawa couplings at small mass

two. At each site of this lattice, there will be an $\mathbf{s} \in \Omega$, the position of which will be denoted by a Greek superscript to specify the layer and a Latin subscript to denote the position in $\mathbb{Z}^{d}$. Thus, in certain generality, one may write

$$
-\mathcal{H}=\sum_{\substack{i, j \in \mathbb{Z}^{d} \\ 1 \leq \alpha, \delta \leq L}} J_{i, j}^{\alpha, \delta}\left(\mathbf{s}_{i}^{\alpha} \cdot \mathbf{s}_{j}^{\delta}\right) .
$$

Normally, the interaction in Eq. (2.7) does not connect the top and bottom of the layer; i.e. there is not an $\mathrm{L}+1^{\text {st }}$ layer which gets identified with the first layer.

For mean-field study, the (finite subsets of) $\mathbb{Z}^{d}$ become the complete graph of $N$ sites, that is each $J_{i, j}^{\alpha, \delta}$ becomes independent of $i$ and $j$ and gets scaled by $N^{-1}$ :

$$
J_{i, j}^{\alpha, \delta} \rightarrow \frac{J}{N} Q^{\alpha, \delta}
$$

The $(\alpha, \delta)$ dependence therefore represents a coupling between layers each of which acts as a mean-field system. The simplest non-trivial model, namely

$$
Q^{\alpha, \delta}= \begin{cases}1 ; & \text { if } \alpha=\delta \\ \gamma ; & \text { if }|\alpha-\delta|=1 \\ 0 ; & \text { otherwise }\end{cases}
$$

already captures most of the essential features (which will be discussed below). For the purposes of this note, it will be assumed that $J_{i, j}^{\alpha, \delta}$ is of the form

$$
J_{i, j}^{\alpha, \delta}=J_{i, j}(\mu) Q^{\alpha, \delta},
$$


where $J_{i, j}(\mu)$ is of the Yukawa form that is in Eq. (2.2) and $Q^{\alpha, \delta}$ is a symmetric, positive definite matrix. The motivation for the above restriction - more generality is certainly possible - will be clear from the following proposition the proof of which is immediate and will be presented immediately.

Proposition 2.2. For single spin-space $\Omega$ (which is a subset of a finite-dimensional vector space with positive definite inner product) consider the layered model with $J_{i, j}^{\alpha, \delta}=$ $J_{i, j}(\mu) Q^{\alpha, \delta}$, where $J_{i, j}(\mu)$ is of the Yukawa form (or any other reflection positive pair interaction) and $Q$ is positive definite. Then the interaction for the layered system as described by the Hamiltonian in Eq. (2.7) is reflection positive with respect to all the standard $\mathbb{Z}^{d}$ reflections in planes between sites (i.e. in those $\{1, \ldots, \mathrm{L}\} \times \mathbb{Z}^{d-1}$ hyper-plane segments with normals orthogonal to the layering direction).

Proof. The idea is to write the model as a reflection positive model on $\mathbb{Z}^{d}$ from which the result follows immediately. To this end, the spin-space will be $\Omega^{L}$ and if $\mathbb{S}$ and $\mathbb{G}$ are "spins" in $\Omega^{\perp}$ with $\mathbb{S}=\left(\mathbf{s}^{1}, \ldots \mathbf{s}^{\mathrm{L}}\right)$ and $\mathbb{G}=\left(\mathbf{g}^{1}, \ldots \mathbf{g}^{\mathrm{L}}\right)$ we define

$$
((\mathbb{S} \circ \mathbb{G}))=\sum_{\alpha, \delta} Q^{\alpha, \delta}\left(\mathbf{s}^{\alpha} \cdot \mathbf{g}^{\delta}\right) .
$$

It is now is enough to demonstrate that $((\cdot \circ \cdot))$ is a positive definite inner product on $\Omega^{L}$. To see this, we simply write, for any $\mathbb{S} \in \Omega^{L}$, the relevant expression: $((\mathbb{S} \circ \mathbb{S}))=$ $\sum_{\alpha, \delta} Q^{\alpha, \delta}\left(\mathbf{s}^{\alpha} \cdot \mathbf{s}^{\delta}\right)$. Since the original inner product on $\Omega$ is positive definite, we may express $\left(\mathbf{s}^{\alpha} \cdot \mathbf{s}^{\delta}\right)=\sum_{n} \lambda_{n} c_{n}^{\alpha} c_{n}^{\delta}$ with $\lambda_{n}>0$ and the demonstration is completed, by an exchange of the summations, and by noting the positivity of $Q$.

Remark 3. It is remarked that the above sort of grouping is a device that has been employed before, e.g. in [20] - albeit with some extra restrictions. In addition it is noted, without proof, that non-mean-field interactions down the chain can be immediately incorporated into the above formalism by declaring this to be part of the "singlespin measure" on $\Omega^{L}$. Indeed, this will form the basis for some analyses of quantum spin-systems in a future publication.

The layered systems of interest have $Q$ of the form in Eq. (2.9) and the generalization that $Q^{\alpha, \delta}=\gamma_{\ell}$ if $|\alpha-\delta|=\ell$ with $1<\ell<\mathrm{L}$ and it may be assumed, for simplicity, that each $\gamma_{\ell} \geq 0$. It is reemphasized that in the layering direction, the coupling is not to be periodically continued. The interaction matrix may be rewritten in the form $Q=(1+2 \gamma) 11+\gamma \Delta_{\gamma}$ with

$$
\gamma=\sum_{\ell} \gamma_{\ell}
$$

and $\Delta_{\gamma}$ the form of a generalized 1D Laplacian. The eigenvalues of this Laplacian are of the form $-\lambda_{k^{\prime}}$, where

$$
\lambda_{k^{\prime}}=\frac{1}{\gamma} \sum_{\ell} 2 \gamma_{\ell}\left[1-\cos \ell \pi\left(\frac{k+1}{L+1}\right)\right]
$$

with $k^{\prime}=0,1, \ldots L-1$ and, under most circumstances, $k^{\prime}=k$. Notice, then that the matrix $Q$ is positive definite if and only if $1+\sum_{\ell} 2 \gamma_{\ell} \cos \frac{\ell(k+1) \pi}{\mathrm{L}+1}>0$ for all $k$; a condition that shall be henceforth assumed. Let $\lambda_{0}$ denote the magnitude of the smallest 
eigenvalue. Then, as was shown in [10] there is a magnetic transition in the mean-field layered systems. Indeed, in the Ising system, at temperature parameters greater than that given by

$$
\beta_{c ; \mathrm{I}}^{(\mathrm{L})}=\left(1+2 \gamma-\gamma \lambda_{0}\right)^{-1}
$$

the magnetization profile is not-trivial and satisfies the mean-field equation

$$
m_{\alpha}=\tanh \left(\beta\left[(1+2 \gamma) m_{\alpha}+\gamma \Delta_{\underline{\gamma}} m_{\alpha}\right]\right)
$$

where $m_{\alpha}$ magnetization is on the $\alpha^{\text {th }}$ layer. The situation for the XY-model is similar with

$$
\beta_{c ; \mathrm{XY}}^{(\mathrm{L})}=\frac{1}{2}\left(1+2 \gamma-\gamma \lambda_{0}\right)^{-1}
$$

and the magnetization profile satisfying

$$
m_{\alpha}=\frac{I_{1}\left(\beta\left[(1+2 \gamma) m_{\alpha}+\gamma \Delta_{\underline{\gamma}} m_{\alpha}\right]\right)}{I_{0}\left(\beta\left[(1+2 \gamma) m_{\alpha}+\gamma \Delta_{\underline{\gamma}} m_{\alpha}\right]\right)},
$$

where the I's are modified Bessel functions. It may be presumed that under most circumstances, the smallest eigenvalue corresponds to $k=0$. (A sufficient condition, for large $\mathrm{L}$, is that only a finite number of $\gamma$ 's are non-zero and that $\gamma_{1}$ is large compared to all the others.) Under these (and perhaps other) restrictions, one may compute

$$
\lambda_{0} \approx \frac{\pi^{2}}{\mathrm{~L}^{2}} \frac{1}{\gamma} \sum_{\ell} \gamma_{\ell} \ell^{2} .
$$

It is noted, by a variety of arguments, that the "bulk" transition temperature is simply the $\mathrm{L} \rightarrow \infty$ limit of the formulas in Eqs. (2.14)-(2.16). Thus, there is a shift in the transition temperature which, written in reduced form, is given by

$$
\frac{\beta_{c ; \mathrm{I}}^{(\mathrm{L})}-\beta_{c ; \mathrm{I}}^{(\infty)}}{\beta_{c ; \mathrm{I}}^{(\infty)}}=\frac{\gamma \beta_{c ; \mathrm{I}}^{(\infty)} \lambda_{0}}{1-\gamma \beta_{c ; \mathrm{I}}^{(\infty)} \lambda_{0}} \approx \gamma \beta_{c ; \mathrm{I}}^{(\infty)} \lambda_{0}
$$

and similarly for the XY, where the approximate statement is, e.g. for $\mathrm{L}$ large under the assumption that Eq. (2.18) is valid. This is a quantitative version of the qualitative results for the temperature shift found in the aforementioned physics papers. So far at the level of Eqs. (2.14)-(2.16) - this is exact only in the context of the mean-field theory. Later, in Theorem 4.6 we shall see that these formulas are of some pertinence to the actual systems (with Yukawa style interactions). 


\section{Statement of Results}

While all the results of this note concerning phase transitions pertain to $\mathbb{Z}^{d}$ with $d \geq 2$, most of the results of interest are confined to the $2 \mathrm{D}$ cases. Indeed, as mentioned in the Introduction, essentially all of these results can be proved by other methods in $d \geq 3$. Moreover, for cases of transitions associated with the breaking of continuous symmetries, some of the results, e.g. concerning local observables, are trivial since these symmetries are also broken in the actual systems when $d \geq 3$. Nonetheless, only small additional effort is required for $d \geq 3$ so the extra generality will be retained.

The main general result of this note is

Main Theorem. Let $\mathcal{H}$ denote a Yukawa Hamiltonian of the form described in Eqs. (2.1)(2.2) on $\mathbb{Z}^{d}$ with $d \geq 2$ and suppose that the associated mean-field theory has a Generic First Order Scenario at temperature parameter $\beta_{t}^{\mathrm{MF}}$. Then, for $\mu$ sufficiently small, the $d$-dimensional system also has a first order transition at a parameter $\beta_{t}$ which is near $\beta_{t}^{\mathrm{MF}}$. In particular, the transition becomes asymptotically close to its mean-field description in the sense that (1) $\beta_{t} \rightarrow \beta_{t}^{\mathrm{MF}}$ and (2) on both sides of the transition, the block magnetizations averaged over block regions of scale $\ell_{0}$, where $\ell_{0}$ is large compared with unity but small compared with $\mu^{-1}$ can only take on values assymptotically close to the permitted values that are predicted by mean-field theory.

In the cases where certain phases of the model may be characterized by a broken symmetry, we have

Proposition 3.1. Let $\mathcal{H}$ denote a d-dimensional Yukawa Hamiltonian of the form described in Eqs. (2.1)-(2.2) with $d \geq 2$ and suppose that the model has a "discrete symmetry" meaning that there is a group, $\mathbb{A}$, of linear maps $\{A: \Omega \rightarrow \Omega \mid A \in \mathbb{A}\}$ which are measure preserving bijections and are also isometric (with respect to the inner prod$u c t)$. Further suppose, in the context of the associated mean-field theory, that $\mathfrak{M}_{\beta}$ may be decomposed into $k$ (with $k$ finite) disjoint separated convex sets $\mathfrak{M}_{\beta}=\cup_{j=1}^{k} M^{(j)}$ such that $\mathbb{A}$ acts transitively on $\left\{M^{(1)}, \ldots, M^{(k)}\right\}$. Then, for all $\mu$ sufficiently small, the model exhibits (as many as) $k$ distinct phases characterized by global magnetizations close to the values in $M^{(j)}$. In particular, under these conditions, in any shift invariant ergodic Gibbs state derived from this interaction, the magnetization is in the vicinity of one of these sets.

In many cases of interest, the first order phase transition may be from a symmetric state into a phase of broken symmetry - sometimes described as a transition featuring the spontaneous breaking of symmetry. For these cases, and certain generalizations, we have

Theorem 3.2. Let $\mathcal{H}$ denote a d-dimensional Yukawa Hamiltonian of the form described in Eqs. (2.1)-(2.2) with $d \geq 2$. Suppose that the associated mean-field theory has a Generic First Order Scenario at some $\beta_{t}^{\mathrm{MF}}$ and that there is a symmetry group $\mathbb{A}$ for the model of the sort described in Proposition 3.1. Here is is supposed that $\mathfrak{M}_{\beta_{t}^{\mathrm{MF}}}=M_{I} \cup M_{I I}$ with $M_{I}$ and $M_{I I}$ separated and each the union of disjoint separated convex sets, e.g. $M_{I}=\left\{M_{I}^{(1)}, \ldots M_{I}^{\left(k_{I}\right)}\right\}$, on which $\mathbb{A}$ acts transitively and that this description holds, for the sets $\mathbb{M}_{I}$ and $\mathbb{M}_{I I}$, throughout the range $\left[\beta_{I}, \beta_{I I}\right]$. Then, for all $\mu$ sufficiently small, at a value of $\beta$ near $\beta_{t}^{\mathrm{MF}}$, the model exhibits (as many as) $k_{I}+k_{I I}$ distinct phases, $k_{I}$ of them characterized by global magnetizations close to the values in $M_{I}^{(j)}$, 
etc. Furthermore, throughout the above mentioned range of $\beta$, in any shift invariant ergodic Gibbs state derived from this interaction, the magnetization is in the vicinity of one of the above mentioned sets.

Moreover, and of greater substance: For general, $\mathcal{M}_{\beta_{t}^{\mathrm{MF}}}=M_{I} \cup M_{I I}$ under "only" the hypotheses that for every $m_{I} \in M_{I}$ and $m_{I I} \in M_{I I}$,

$$
m_{I I}^{2} \geq m_{I}^{2}+\frac{2}{\beta_{t}^{\mathrm{MF}}} \Delta_{\mathcal{E}}
$$

for some $\Delta_{\mathcal{E}}>0$, then the model, in fact, has a Generic First Order Scenario. Thus if the detailed structure of $M_{I}$ and $M_{I I}$ is as described in the first paragraph, all of the above conclusions hold along with the obvious necessity that this transition is accompanied by a discontinuity in the energy density. Moreover the gap that is not much smaller than (but perhaps larger than) $\Delta_{\mathcal{E}}$.

Finally, in cases of a system with degeneracies which are not related by symmetry, the following result for the phase diagram will be established:

Theorem 3.3. Let $\mathcal{H}$ denote a d-dimensional Yukawa Hamiltonian of the form described in Eqs. (2.1)-(2.2). Suppose that at parameter $\beta$, in the associated mean-field theory $\mathcal{M}_{\beta}$ consists of $k>1$ (non-trivial) interior points $\left\{m_{1}, \ldots m_{k}\right\}$ of $\mathscr{C}_{\Omega}$ which, considered as elements of $\mathbb{E}_{\Omega}$, are linearly independent. If $b \in \mathbb{E}_{\Omega}$, we may consider the Hamiltonian augmented by the external field $b$ as described subsequent to Eq. (2.1):

$$
-\beta \mathcal{H} \rightarrow-\beta \mathcal{H}+\sum_{i}\left(b \cdot \mathbf{s}_{i}\right)
$$

Then, for any pair $p$ and $q, 1 \leq p<q \leq k$, there is a one-parameter family of fields $b_{\lambda}^{p, q}$, where $-1 \leq \lambda \leq+1$ with $\sup _{\lambda}\left\|\bar{b}_{\lambda}^{p, q}\right\| \rightarrow 0$ as $\mu \rightarrow 0$ such that for some $\lambda \in(-1,+1)$ there is coexistence between two phases with magnetizations near $m_{p}$ and $m_{q}$ respectively. Furthermore for all $\lambda \in[-1,+1]$ at the level of block observables, (on regions that are large compared with unity but small compared with $\mu^{-1}$ ) all the other values of magnetizations are suppressed with high probability.

Remark 4. It is remarked that in the context of spin-systems, linear independence of the spin-states and/or the constituent sets of $\mathcal{M}_{\beta_{t}^{\mathrm{MF}}}$ is not a common occurrence in the systems that are usually studied. Indeed for a spin-system there is a physical $\Omega$ which, perhaps, is endowed with 'internal' symmetries that are natural to the problem at hand. The dimension of $\Omega$ may be vastly smaller than the actual or effective number of spin-states, but this is where the symmetries come into play. As a consequence of these symmetries, often enough, it is sufficient to align the external field with the desired state to select this state among all others related by symmetry. Then, even for weak fields, this will alter the nature phase diagram and the associated transitions. Well known examples include the $q$-state Potts model and the $r$-cubic model where, in the field-temperature plane, generic first order scenarios can be established with phase transitions at non-zero field which are markedly different from those in zero field. However, these sorts of systems - which do not satisfy the linear independence hypotheses of Theorem 3.3 but compensate by having a sufficient degree of internal symmetry - seem difficult to classify under a general principle. Thus, while it is clear that many particular results on in-field transitions can be established with the present methods, here, for the sake of brevity, we 
shall refrain from any specific claims. Indeed the Potts model in an external field was analyzed in [3] which, it seems, required a certain degree of effort.

In the context of this work, the pertinent cases for Theorem 3.3 are particle systems where the different spin-states represent different particle types. There is no actual $\Omega$ in the problem and thus one has to be constructed. Geometrically the simplest - and in a certain sense the most realistic - possibility is mutually orthogonal states in $\mathbb{R}^{n}$ where $n$ corresponds to the number of species, along with some non-diagonal interaction. Here, of course, the external fields represent activities for the various species (albeit with the Euclidean notion of inner product, which is presumably not the same as the inner product defining the particle-particle interactions). Under these circumstances it is most plausible that degenerate minima of $\Phi_{\beta}$, now representing excesses of various species, will indeed end up linearly independent.

\section{Results for Specific Systems}

In this section, specific examples will be provided for the various phenomena alluded to previously. Foremost:

- Discrete spin-systems with symmetry. The best known example is the $q$-state Potts model where, as is often the convention, each $\operatorname{spin} \mathbf{s}_{i}$ is taken to point to a vertex of a $(q-1)$-dimensional hypertetrahedron and the inner product is defined by the usual Euclidean dot product. Hence $\mathbf{s}_{i} \cdot \mathbf{s}_{j}$ is essentially given by a Kronecker delta. On the basis of Proposition 3.1 and Theorem 3.2 along with some analysis of the mean-field theory (most of which was done in [2]) the following is established:

Theorem 4.1. Consider the q-state Potts version of the Hamiltonian described in Eqs. (2.1)-(2.2) on $\mathbb{Z}^{d}$ with $d \geq 2$ and with $q \geq 3$. Then for $\mu^{-1}$ large, there is a first order transition at some $\beta_{t}$ featuring (at least one) high temperature state with small or vanishing magnetization and (at least) $q$ low temperature states characterized by substantial magnetization in the different hypertetrahedral directions. Furthermore, this transition is accompanied by a discontinuity in the energy density. For $\beta>\beta_{t}$, the high temperature state disappears while the low temperature states persist, while for $\beta<\beta_{t}$, the low temperature phases are not present. Finally, the value of $\beta_{t}$ as well as the free energy, magnetization and energy density at and beyond $\beta_{t}$ are (at least in some neighborhood of $\beta_{t}$ ) uniformly close to the appropriate mean-field formulas, e.g. as appear in [34].

Less well known but also of interest are the cubic models in which each spin $\mathbf{s}_{i}$ points to the face of an $r$-dimensional cube and one again employs the usual Euclidean inner product. Here the result is

Theorem 4.2. Consider the r-cubic version of the Hamiltonian described in Eqs. (2.1)(2.2) on $\mathbb{Z}^{d}$ with $d \geq 2$ and with $r \geq 4$. Then for $\mu^{-1}$ large, there is a first order transition at some $\beta_{t}$, which features coexistence between $2 r$ low temperature states and a high temperature state and a discontinuity in the energy density. The properties of these states are similar, after appropriate modifications, to those described for the Potts model in the statement of Theorem 4.1 .

- Phase coexistence in models without symmetry. The vast majority of realistic lattice gasses fall into this category - there is no anticipation of symmetries as there would be in a spin-system. Indeed, under these auspices, the range of possible models and their 
possible modes of behavior is so vast that the general situation is overwhelming. Thus we shall be content with a single example which, in the opinion of the author, could not easily be treated by other methods. Consider, then, a tertiary alloy - a lattice gas with three species $a, b$ and $c$. It is assumed that each site $i$ is occupied by one of the three species; thus we have the variables $\eta_{i}^{a}, \eta_{i}^{b}$ and $\eta_{i}^{c}$ each in $\{0,1\}$ with $\eta_{i}^{a}+\eta_{i}^{b}+\eta_{i}^{c}=1$. It will be stipulated that each species has a pair interaction with strengths $J_{a}>J_{b}>J_{c}-$ so that species $b$ and $c$ will be suppressed, more heavily the latter. As "compensation" there will be repulsion between species $a$ and $b$ and an attraction between $b$ and $c$. The (formal) Hamiltonian for the model is therefore given by

$$
-\beta \mathcal{H}=\sum_{i, j}\left[J_{i, j}^{a} \eta_{i}^{a} \eta_{j}^{a}+J_{i, j}^{b} \eta_{i}^{b} \eta_{j}^{b}+J_{i, j}^{c} \eta_{i}^{c} \eta_{j}^{c}-K_{i, j}^{a b} \eta_{i}^{a} \eta_{j}^{b}+K_{i, j}^{b c} \eta_{i}^{b} \eta_{j}^{c}\right]
$$

In the above, the notation for couplings has been defined so that all the $K$ 's and $J$ 's are non-negative. Here, we have the following:

Theorem 4.3. Consider the Hamiltonian defined in Eq. (4.1) with couplings given by the Yukawa form in Eq. (2.2) without the specific normalization condition. Let $J_{a}$ denote the sum $J_{a}=\sum_{i} J_{0, i}^{a}$ and similarly for $J_{b}, \ldots, K_{b c}$. Let us express $J_{a}=J+D_{a}$, $J_{b}=J+D_{b}$ and $J_{c}=J$. Then the following holds for all $\mu$ sufficiently small: For $J$ sufficiently large, $K_{a b}, K_{b c}$ comparatively (sufficiently) small and $D_{a}, D_{b}$ (sufficiently) smaller still, there is a point $K^{\star}=\left(K_{a b}^{\star}, K_{b c}^{\star}\right)$ - both components positive - such that the Hamiltonian augmented with various (natural) activities exhibits phase coexistences between pairs among three types of phases at certain values of the activities. The three phase types are characterized by dominance of one of the species over the other two. Furthermore, as $\mu \rightarrow 0$, the requisite activities for coexistence tend to zero.

- Low temperature behavior for low-D models with continuous symmetry. Here we shall state the formal results for models with $O(n)$ symmetry; see the paragraph following the proof of Corollary 5.2 for further discussion.

Theorem 4.4. Consider the standard $O(n)$ spin-system with $n \geq 2$, i.e. each $\mathbf{s}_{i}$ is an $n$-dimensional unit vector and the inner product is the usual Euclidean dot product with the Yukawa interaction (Eqs. (2.1)-(2.2)) in $d \geq 1$. Let $\beta \approx \beta_{c}^{\mathrm{MF}}=n$. Then, for all $\mu$ sufficiently small, there is a scale $\ell_{0}$ (which tends to infinity as $\mu \rightarrow 0$ ) such that on any compact interval of temperatures, the spatially averaged magnetization at this scale (cf. Eq. (5.1)) is, with high probability, uniformly close to $m_{M F}(\beta) \hat{v}$, where $\hat{v}$ is an $n-$ dimensional unit vector and $m_{M F}$ is a solution of the mean-field equation $m=m_{n}(\beta m)$, where $\mathrm{m}_{n}(h)$ is the scalar magnetization function:

$$
\mathrm{m}_{n}(h)=\frac{\int_{-1}^{+1} e^{h x}\left(1-x^{2}\right)^{\frac{n-3}{2}} x d x}{\int_{-1}^{+1} e^{h x}\left(1-x^{2}\right)^{\frac{n-3}{2}} d x} .
$$

Moreover the energy and free energy per spin is uniformly close to the appropriate mean-field formula.

The $O(n)$ nematic models are most easily described in the context of the $O(n)$ spinsystems with the pair interaction between spins at sites $i$ and $j$ replaced by $\left(\mathbf{s}_{i} \cdot \mathbf{s}_{j}\right)^{2}$. Note that for $n=2$ this is, for all intents and purposes, equivalent to an XY spin-system; but 
not so for $n \geq 3$ which will henceforth be assumed. For a variety of reasons (not all of which are understood by the author) this is always presented via the traceless matrices

$$
K^{p, q}=\mathbf{s}^{(p)} \mathbf{s}^{(q)}-\frac{1}{n} \delta^{p, q},
$$

where $\mathbf{s}^{(p)}$ denotes the $p^{\text {th }}$ component of an $O(n)$ spin. Then, the pair interaction between the spins at sites $i$ and $j$ is given by $\operatorname{Tr}\left(K_{i} K_{j}\right)$. In any case the device of using symmetric traceless $n \times n$ matrices with this notion of inner product and with an a priori measure given by the pullback from the unit sphere in $\mathbb{R}^{n}$ has the advantage that it constitutes the ingredients for a bona fide mean-field theory. Since this mean-field theory was the subject of a good deal of analysis in [2], the relevant results will be summarized briefly:

(a) For all $\beta$, the minimizing $K$ are orthogonally equivalent to a diagonal matrix of the form

$$
K=\lambda(\beta) \operatorname{diag}\left[1,-\frac{1}{n-1}, \ldots,-\frac{1}{n-1}\right],
$$

where $\lambda$ is a solution of the mean-field equation

$$
\lambda=\frac{\int_{0}^{1} e^{\beta^{\prime} \lambda x^{2}}\left(1-x^{2}\right)^{\frac{n-3}{2}}\left(x^{2}-\frac{1}{n}\right) d x}{\int_{0}^{1} e^{\beta \lambda \lambda x^{2}}\left(1-x^{2}\right)^{\frac{n-3}{2}} d x}
$$

with $\beta^{\prime}=\left(1-\frac{1}{n}\right) \beta$. Indeed all local minima of the free energy function have this property.

(b) There is a $\beta_{t}^{\mathrm{MF}}$ such that for $\beta \leq \beta_{t}^{\mathrm{MF}}, \Phi_{\beta}(K)$ is minimized by $K \equiv 0$ while for $\beta \geq \beta_{t}^{\mathrm{MF}}, \Phi_{\beta}(K)$ is minimized by a non-trivial $K$ as described in (a) with some $\lambda(\bar{\beta}) \geq \lambda\left(\beta_{t}^{\mathrm{MF}}\right)=\lambda_{t}^{\mathrm{MF}}>0$.

Thus we see a standard mean-field type of first order transition featuring coexistence of states with differing energy that is accompanied by the breaking of a continuous symmetry. Here we shall prove:

Theorem 4.5. Consider an $O(n)$ nematic spin-system in $d \geq 2$ with $n \geq 3$ as described above (spins $K_{i}$ are $n \times n$ symmetric traceless matrices, the inner product given by $\operatorname{Tr}\left(K_{i} K_{j}\right)$ and $\alpha_{0}$ the pullback of Haar measure on the unit $n$-dimensional sphere; or the simpler description with the usual unit n-dimensional spins and the pair interactions defined by the square of the Euclidean dot product) with couplings as given in Eq. (2.2). Then, for all $\mu$ sufficiently small, there is a $\beta_{t}(\mu)$ with $\beta_{t} \rightarrow \beta_{t}^{\mathrm{MF}}$ such that at $\beta_{t}$ (at least) two states coexist; one, a high-temperature state where the energy is small and the other where the energy is substantial. Moreover, in the latter there is a scale $\ell_{0}$, where $\ell_{0} \gg 1$ if $\mu^{-1}$ is large such that within blocks of this scale, the spatially averaged nematic-spin variable is, with high probability, of the form in Eq. (4.4) or an orthogonal transformation thereof. In the high temperature state, and for all $\beta<\beta_{t}$ the spatial average at scale $\ell_{0}$ is close to zero. For $\beta>\beta_{t}$, on any compact interval, there is a $\mu_{0}$ such that for all $\mu<\mu_{0}$ the statement concerning the low temperature state holds for $\beta>\beta_{t}$.

- Low temperature results for the layered Ising and XY models. Here some preliminary results for the layered systems are presented. More general results for the continuous spin models especially in dimension greater than two are possible but are not of immediate physical relevance and so will be omitted. 
Theorem 4.6. Consider layered Ising and XY on $\mathbb{Z}^{d} \times\{1, \ldots \mathrm{L}\}$ as described by the interactions in Eqs. (2.10) and (2.1)-(2.2), where $Q$ is as described just prior to Eq. (2.12) with each $\gamma_{\ell}$ non-negative. Then for $\beta$ less than the formulas given in Eqs. (2.14) and (2.16) respectively, the magnetization vanishes. Moreover, for all $\mu$ sufficiently small, there is a scale $\ell_{0}$ (which can tend to infinity as $\mu \rightarrow 0$ ) such that on any compact interval of temperatures, the block averaged magnetization profiles, free energies and energies are uniformly close to those given by the appropriate mean-field formulas. Finally, in the case of the Ising version, the global magnetization profile agrees closely with the block magnetization as just described.

\section{Proofs}

5.1. General properties: magnetics. Most of the results stated in Sect. 3 are a direct consequence of the following:

Lemma 5.1. Consider the spin-system defined by the interaction in Eq. (2.1) with couplings as in Eq. (2.2) at interaction parameter $\mu$ defined on the d-dimensional toroidal lattice. For an integer $\ell_{0}$, let $\Lambda_{\ell_{0}}$ denote a cube of side length $\ell_{0}$ and let

$$
\mathfrak{m}_{\ell_{0}}=\frac{1}{\left|\Lambda_{\ell_{0}}\right|} \sum_{i \in \Lambda_{\ell_{0}}} \mathbf{s}_{i}
$$

denote the block magnetization of $\Lambda_{\ell_{0}}$, and let us assume for simplicity that the linear dimension of the torus, $L$, is of the form $L=2^{k} \ell_{0}$. Let $m \in \operatorname{Conv}(\Omega)$, and if $\Delta>0$ is a real number let $\mathcal{N}_{\Delta}(m)$ denote the neighborhood ball of radius $\Delta$ about m. Let $\mathbb{K}_{\mathcal{N}_{\Delta}}^{\left[\ell_{0}\right]}(m)$ denote the event

$$
\mathbb{K}_{\mathcal{N}_{\Delta}}^{\left[\ell_{0}\right]}(m)=\left\{\mathfrak{m}_{\ell_{0}} \in \mathcal{N}_{\Delta}(m)\right\}
$$

Then there is an $\epsilon=\epsilon\left(\ell_{0}, \mu, \Delta\right)$ with the explicit bound

$$
\epsilon<\epsilon_{0}=\beta \omega \Delta+\frac{1}{2} \beta \Delta^{2}+c \mu \ell_{0} \beta \omega^{2}
$$

where $c$ is a uniform constant of order unity and

$$
\omega=\sup \{m \mid m \in \operatorname{Conv}(\Omega)\}
$$

such that as $L \rightarrow \infty$,

$$
\varlimsup \alpha_{\beta, \mathbb{T}_{L}}\left(\mathbb{K}_{\mathcal{N}_{\Delta}}^{\left[\ell_{0}\right]}(m)\right) \leq e^{-\left(\Phi_{\beta}^{\mathcal{N} \Delta}(m)-F_{\mathrm{MF}}(\beta)-\epsilon\right) \ell_{0}^{d}},
$$

where, in the above

$$
\Phi_{\beta}^{\mathcal{N}_{\Delta}}(m)=\inf _{m^{\prime} \in \mathcal{N}_{\Delta}^{\prime}(m)} \Phi_{\beta}\left(m^{\prime}\right)
$$


Proof. By the standard chessboard estimates,

$$
\alpha_{\beta, \mathbb{T}_{L}}\left(\mathbb{K}_{\mathcal{N}_{\Delta}}^{\left[\ell_{0}\right]}(m)\right) \leq\left[\frac{Z_{L, \beta}^{\frac{1}{L^{d}}}\left(\mathbb{K}_{\mathcal{N}_{\Delta}}^{\left[\ell_{0}\right]}(m)\right)}{Z_{L, \beta}^{\frac{1}{L^{d}}}}\right]^{\ell_{0}^{d}},
$$

where $Z_{L, \beta}\left(\mathbb{K}_{\mathcal{N}_{\Delta}}^{\left[\ell_{0}\right]}(m)\right)$ is the partition function which is constrained so that in each translate of $\Lambda_{\ell_{0}}$ by a vector with integer multiples of $\ell_{0}$ as components, the translate of the event $\mathbb{K}_{\mathcal{N}_{\Delta}}^{\left[\ell_{0}\right]}(m)$ occurs. (Arguments of this sort are found in the classic papers on reflection positivity. All relevant results for this work can be found in [30]; the interested reader is also referred to the recent review [1] where these sorts of arguments and some new extensions are well explained. Within the above two reviews, all the necessary references can be found.) Therefore let us seek upper bounds along the lines of $\sim e^{-\Phi_{\beta}(m) L^{d}}$ on the constrained partition function and lower bounds of the form $\sim e^{-F_{\mathrm{MF}} L^{d}}$ on the full partition function. A proof of the latter can be found in the beginning of Theorem 1.1 in [2] although other versions of this result are part of the classic literature on the subject; see, e.g. the book [32] Sect. II.13 - II.14. In any case, we have that

$$
Z_{L, \beta}^{1 / L^{d}} \geq e^{-F_{\mathrm{MF}}+g L^{-1}}
$$

with $g$ a constant.

Turning to the necessary upper bound, let us begin with an estimate of the energetic contribution to the partition function under the above mentioned constraints. In particular, we will show that under this constraint, the total energy is approximately $-\frac{1}{2} \beta m^{2}$ times the volume. (Fortunately, it turns out, the ensuing estimate does not depend on the details of how the constraints are satisfied. Moreover the bounds are nearly optimal in the sense that a similar derivation produces a lower bound which does not differ by too much.) Consider two blocks, which are appropriate translates of $\Lambda_{\ell_{0}}$, that are labeled $V_{p}$ and $V_{q}$ respectively. Let us define the average coupling

$$
\mathbf{J}_{p, q}=\frac{1}{\left|\Lambda_{\ell_{0}}\right|^{2}} \sum_{\substack{i \in V_{p} \\ j \in V_{q}}} J_{i, j}
$$

For generic $i \in V_{p}, j \in V_{q}$, we may write $J_{i, j}=\mathbf{J}_{p, q}\left(1+\kappa_{i, j}\right)$, however, it is clear that under the condition $\mu \ell_{0} \ll 1$, the $\kappa_{i, j}$ are small. Indeed since no two points in $V_{p}$ differ by more than the order of $\ell_{0}$ - and similarly for points in $V_{q}$ - from Eq. (2.2), it is easily seen that

$$
\left|\kappa_{i, j}\right| \leq 2 c \mu \ell_{0}
$$

where $c$ depends on dimension - but not on $p$ and $q$-and the two is for convenience. Thus if $m_{p}$ and $m_{q}$ (which satisfy the criterion for the event $\mathbb{K}_{\mathcal{N}_{\Delta}}^{\left[\ell_{0}\right]}(m)$ ) are the magnetizations in their respective blocks, then

$$
\sum_{\substack{i \in V_{p} \\ j \in V_{q}}} J_{i, j}\left(\mathbf{s}_{i} \cdot \mathbf{s}_{j}\right)=\left|\Lambda_{\ell_{0}}\right|^{2} J_{p, q}\left(m_{p} \cdot m_{q}\right)+J_{p, q} \sum_{\substack{i \in V_{p} \\ j \in V_{q}}} \kappa_{i, j}\left(\mathbf{s}_{i} \cdot \mathbf{s}_{j}\right) .
$$


The rightmost term in Eq. (5.9) is relatively small and, further, the quantity $\left(m_{p} \cdot m_{q}\right)$ may be replaced by $\tilde{m}^{2}$ for any $\tilde{m} \in \mathcal{N}_{\Delta}(m)$ at cost of only a small additional error. Thus, all in all - for any $\tilde{m} \in \mathcal{N}_{\Delta}(m)-$

$$
\begin{aligned}
\sum_{\substack{i \in V_{p} \\
j \in V_{q}}} J_{i, j}\left(\mathbf{s}_{i} \cdot \mathbf{s}_{j}\right) & \leq J_{p, q}\left|\Lambda_{\ell_{0}}\right|^{2}\left[\tilde{m}^{2}+2 \Delta \omega+\Delta^{2}+2 c \mu \ell_{0} \omega^{2}\right] \\
& \equiv J_{p, q}\left|\Lambda_{\ell_{0}}\right|^{2}\left[\tilde{m}^{2}+\frac{2}{\beta} \epsilon\right] .
\end{aligned}
$$

It has been assumed that $p \neq q$; a similar-minded argument with corresponding results may be obtained for the "diagonal" terms - or these may be neglected altogether in the small $\mu$ limit. In any case, summing Eq. (5.10) over all pairs, (and noting the normalization condition on the $J_{i, j}$ described after Eq. (2.2)) the anticipated estimate for the energetics has been obtained.

Let us turn to the entropic considerations. Since each cube in the torus acts independently, the term to be estimated is simply the appropriately constrained $\alpha_{0}$-measure of the spin-configurations in $\Lambda_{\ell_{0}}$. It is claimed that there is some $m^{\sharp} \in \mathcal{N}_{\Delta}(m)$ such that

$$
\int\left[\prod_{j \in \Lambda_{\ell_{0}}} d \alpha_{0}\left(\mathbf{s}_{j}\right)\right]\left[\mathbb{I}_{\left\{\mathfrak{m}_{\ell_{0}} \in \mathcal{N}_{\Delta}(m)\right\}}\right] \leq e^{S\left(m^{\sharp}\right)\left|\Lambda_{\ell_{0}}\right|} .
$$

The derivation is as follows (assuming that the left-hand side is not trivial): Since, conditionally with probability one, $\mathfrak{m}_{\ell_{0}} \in \mathcal{N}_{\Delta}(m)$, then the average of $\mathfrak{m}_{\ell_{0}}$, denoted by $m \sharp$ is also in $\mathcal{N}_{\Delta}(m)$. Let $A_{\ell_{0}}, \mathcal{N}_{\Delta}$ denote the normalized measure corresponding to the constrained product measure. Then, by Jensen's inequality,

$$
\int d A_{\ell_{0}, \mathcal{N}_{\Delta}} e^{\sum_{j}\left(\mathbf{s}_{j} \cdot h\right)} \geq e^{\left|\Lambda_{\ell_{0}}\right|\left(h \cdot m^{\sharp}\right)},
$$

i.e.

$$
\begin{aligned}
& \int\left[\prod_{j \in \Lambda_{\ell_{0}}} d \alpha_{0}\left(\mathbf{s}_{j}\right) e^{\sum_{j}\left[\left(\mathbf{s}_{j} \cdot h\right)-\left(m^{\sharp} \cdot h\right)\right]}\right]\left[\mathbb{I}_{\left\{\mathfrak{m}_{\ell_{0}} \in \mathcal{N}_{\Delta}(m)\right\}}\right] \\
& \quad \geq \int\left[\prod_{j \in \Lambda_{\ell_{0}}} d \alpha_{0}\left(\mathbf{s}_{j}\right)\right]\left[\mathbb{I}_{\left\{\mathfrak{m}_{\ell_{0}} \in \mathcal{N}_{\Delta}(m)\right\}}\right] .
\end{aligned}
$$

The desired result is obtained by relaxing the constraint on the left and seeking the supremum over $h$. It is noted that in the preceding, some mild use has been made of the fact that $\mathcal{N}_{\Delta}(m)$ is a convex set. If it happens that this neighborhood intersects the complement of $\operatorname{Conv}(\Omega)$ the restricted set is still convex. (Or we may stay with the full set and rely on the fact that the measure provides no weight to the complement of $\operatorname{Conv}(\Omega)$ and that outside of $\operatorname{Conv}(\Omega)$, the free energy is infinite.)

To within the stated error tolerances, the upper bound on $Z_{L, \beta}\left(\mathbb{K}_{\mathcal{N}_{\Delta}}^{\left[\ell_{0}\right]}(m)\right)$ as it now stands picks a particular point in $\mathcal{N}_{\Delta}(m)$ to evaluate $\Phi_{\beta}$. Obviously, this may be replaced with the worst case (perhaps limiting) scenario.

As an immediate corollary, we rule out the possibility of any non-mean-field like magnetizations and extend this latter statement to non-toroidal states. 
Corollary 5.2. Let $\Xi_{\eta}(\beta)$ denote the set of magnetizations such that $\Phi_{\beta}(m)$ is within $\eta$ of $F_{\mathrm{MF}}(\beta)$ and let $\mathbb{B}_{\left[\ell_{0}\right], \eta}$ denote the event that $\mathfrak{m}_{\ell_{0}}$ is in the complementary set, i.e. that the block magnetization in $\Lambda_{\ell_{0}}$ corresponds to a mean-field free energy which is further than $\eta$ from any minimizer. Then, with apologies for the 3 , there is a $K(\eta)$ such that for all $\mu \ell_{0}$ sufficiently small, if $L \rightarrow \infty$ along power of two multiples of $\ell_{0}$,

$$
\varlimsup \alpha_{\beta, \mathbb{T}_{L}}\left(\mathbb{B}_{\left[\ell_{0}\right], 3 \eta}\right) \leq K(\eta) e^{-\eta \ell_{0}^{d}}
$$

Further, if $\alpha_{\beta}(-)$ denotes any shift-invariant infinite-volume Gibbs state corresponding to the Hamiltonian in Eqs. (2.1) - (2.2), then there is a $\delta$ that tends to zero with the right-hand side of Eq. (5.14) such that

$$
\alpha_{\beta}\left(\mathbb{B}_{\left[\ell_{0}\right], 3 \eta}\right)<\delta
$$

Moreover, if $\beta$ ranges over a bounded set, then for a given $\eta$, the above holds uniformly for any fixed pair $\left(\mu, \ell_{0}\right)$ - provided $\mu \ell_{0}$ is sufficiently small.

Proof. Once we establish the result in Eq. (5.14), the one in Eq. (5.15) is a direct application of Theorem 2.5 in [5] in the special case of only one "good" event. First let us prove this for fixed $\beta$. For $m \in \bar{\Xi}_{3 \eta}$, let us find a $\Delta_{m}$ such that

(1) $\mathcal{N}_{\Delta_{m}}(m) \cap \Xi_{\eta}=\emptyset$,

(2) $\epsilon_{0}\left(\ell_{0}, \mu, \Delta_{m}\right)<\eta$.

The ability to achieve the former relies on the continuity of $\Phi_{\beta}$ (see the Appendix) and the latter already relies on $\mu \ell_{0}$ sufficiently small. By compactness, only a finite number, $K(\eta)$ of these are needed and the result follows immediately.

As for the uniformity, let us divide the bounded set of $\beta$ 's (conveniently thought of as an interval) into pieces each of size no more than $\Delta_{\beta}$, where $\frac{1}{2} \Delta_{\beta} \omega^{2} \ll \eta$. Notice that the free energy at any $m \in \operatorname{Conv}(\Omega)$ cannot change by more than this small amount as $\beta$ varies over the piece. Thus, there is ample space between the union of the $\Xi_{\eta}(\beta)$ 's and the union of the $\Xi_{3 \eta}$ 's; items (1) and (2) above can be modified accordingly and the result holds throughout the piece. Since the estimate in Eq. (5.14) depends only on the number, $K$, of sets used, the maximum can be chosen.

Remark 5. There has not been any attempt to provide an optimal scheme for the rate of convergence both here and in the second corollary below. Indeed, it is obvious that the estimates are grossly inefficient. For example, it is clear that the principal contribution to an inequality of the form in Eq. (5.14) should come only from the edge of $\Xi_{3 \eta}$. This could be existentially rectified by a modification of the second condition to allow bigger neighborhoods in regions far away from the minimizer and/or using the large value. However, the upshot would still be existential so nothing practical would have been gained. Indeed, better estimates, if actually required, can always be obtained in the context of specific models where the particulars of $\Phi_{\beta}(m)$ can be brought into play.

The second corollary, namely that as the range of the interaction tends to infinity, the free energy converges to $F_{\mathrm{MF}}$, is also immediately available. It should be remarked that many cases of interest are covered by Theorem II.14.1 in the book [32] (which is in turn based on [29]). Indeed, results of this sort date back to the work of Kac in the early 1960's. 
Corollary 5.3. Consider a spin-system on $\mathbb{Z}^{d}$ with interactions as described in Eqs. (2.1)-(2.2). Let $F_{\mu}(\beta)$ denote the free energy: $\lim _{L \rightarrow \infty} Z_{L, \beta}^{1 /\left|\mathbb{T}_{L}\right|}=e^{-F_{\mu}(\beta)}$. Then $\lim _{\mu \rightarrow 0} F_{\mu}(\beta)=F_{\mathrm{MF}}(\beta)$. Further, if $\beta$ ranges over a bounded set, the convergence is uniform.

Proof. The lower bound on $Z_{L, \beta}$ is already in place. Allowing $\Delta, \ell_{0}, \epsilon=\epsilon\left(\Delta, \mu, \ell_{0}\right)$, etc. to denote their previous meanings (with fewer restrictions), let $K^{\prime}$ denote the number of neighborhoods required to cover the whole space. Then, by chessboard estimates (see, in particular the "subadditivity lemma", Lemma 6.3 in [4]) we have

$$
Z_{L, \beta} \leq\left[\left[K^{\prime}\right]^{\frac{1}{\ell_{0}^{d}}} e^{-\left(F_{\mathrm{MF}}-\epsilon\right)}\right]^{\left|\mathbb{T}_{L}\right|},
$$

and the result follows first by taking $L \rightarrow \infty$ and then $\mu \rightarrow 0$ which allows $\ell_{0} \rightarrow \infty$, taking care of the $K$-term so the $\Delta$ (and hence also the $\epsilon<\epsilon_{0}$ error) can go to zero. Uniformity is established as was done at the end of Corollary 5.2: Most of the above has harmless $\beta$ dependencies; the interesting term, involving the $K^{\prime}$, depends on $\eta$ through $\Delta$, and can be uniformly bounded over the whole range: The term is dispensed with by considering worst case (in $\beta$ ) possibilities.

Discussion/Examples. The above results are disquieting, at least at first glance, since the conclusion is that in any system with spread-out interactions, the local magnetization will (more or less) only take on values permitted by mean-field theory. Let us consider the implications in two principal classes of examples: General one-dimensional systems and 2D systems with continuous symmetries. Needless to say, in both cases, the commonly studied mean-field models have phase transitions that are associated with the singular behavior of the magnetic order parameter. This behavior is obviously not possible in a one-dimensional system with Yukawa interactions. (In particular, such interactions are known to satisfy Dobrushin's criterion for complete analyticity [25].) Notwithstanding, the above tells us that at least locally, but not too locally at length scales $1 \ll \ell \leq \ell_{0} \ll \mu^{-1}$, a one-dimensional system will appear to have undergone a phase transition at around a temperature $T_{\mathrm{MF}}\left(\propto\left[\beta_{t}^{\mathrm{MF}}\right]^{-1}\right)$. So, e.g. in cases of magnetic symmetry breaking, once $T<T_{\mathrm{MF}}$, there must be large patches of ordered phase each approximately magnetized according to mean-field theory but, overall, canceling out. A similar picture holds for phase transitions that are not associated with symmetry breaking e.g. that have an $m(\beta)$ (perhaps with a non-magnetic interpretation) undergoing interesting "discontinuous-like" local behavior at temperatures around $T_{\mathrm{MF}}$. It is remarked that while it is obvious that something along the lines of the above must happen as $T \rightarrow 0$, it is now seen, in the large range limit, that this behavior initiates at around $T_{\mathrm{MF}}$ and occurs in an understandable and controllable fashion. Indeed, it should be mentioned that for the Ising model [7] and the standard $O(2) \& O(3)$ Heisenberg models [6], results along these lines have been obtained previously. However in these instances some particulars of the $O(n)$ with $n=1,2,3$ were exploited and, moreover, a sustained effort was required.

Let us now turn to some interesting $2 \mathrm{D}$ cases, namely magnetic systems - such as $O(n)$ systems with $n \geq 2-$ that have continuous symmetries. On general principles $[19,26]$ and generally provable by the methods of [12], the symmetry cannot be broken; that is to say in an infinite-volume state, the magnetization will vanish. So in these circumstances, it would appear, the situation is on par with the general one-dimensional systems. However, there are two outstanding exceptions. (1) Cases where the mean-field 
model enjoys a discontinuous transition accompanied by a (discontinuous) breaking of the continuous symmetry. Here we will find that the discontinuity persists at the local level signaling a $1^{\text {st }}$ order transition, i.e. phase coexistence. However, at the global level, or even at the level of very large-scale behavior there is no breakdown of the symmetry. (2) The specific case of the 2D-XY (or $O(2)$ ) model, has a phase transition, the Kosterlitz Thouless transition, [14,22] with the low temperature phase featuring power law decay of correlations. It is not hard to show, via correlation inequalities, that this must also be the case for the mean-field like version; the question of whether or not this transition actually occurs in the vicinity of $T_{\mathrm{MF}}$ is under investigation.

5.2. Further properties: energetics. Till now, our attention has been focused on the block observables $\mathfrak{m}_{\ell_{0}}$ (and their translates) and, as the range of the interaction gets large, we have seen that these concentrate near the values of the magnetization that are dictated by the corresponding mean-field theory. A similar result for the energy is a more ambitious endeavor since, ultimately, the magnetization observables are strictly local whereas the energy observables are more diffuse. In particular, the results of this subsection will be of a more technical nature - e.g. some additional hypotheses concerning the mean-field theory will be required. In fact, not all of this section is strictly necessary when the free energy minima are simply isolated points, which is often enough the case. Indeed, under these circumstances, the magnetization simply drags the energy along with it and a part of the labour of this subsection is rendered unnecessary. Therefore, for some, this section may be read lightly without much loss of continuity.

The central result of the subsection amounts to a statement that the actual $(\mu \ll 1)$ systems must have "energetics" close to values corresponding to minima or near minima of $\Phi_{\beta}$. The developments will come about in two stages: The first argument goes via quasilocal energy observables, which holds in full generality.

The second part of the argument involves the energy density itself which, if there is coexistence, will require $d \geq 2$. The latter is, of course, an absolute necessity since, in $d=1$, the energy is continuous and therefore, when the mean-field theory has a discontinuity, the actual system will take on intermediate values. This is brought about by combinations of spatially separated regions which themselves have nearly sharp "allowed" values but are uncorrelated. By contrast, for $d \geq 2$ the above mentioned quasilocal energies maintain a coherence and, as a consequence, the global energy is always near some value corresponding to a minimizer or near minimizer of $\Phi_{\beta}$. This necessitates, above $d=1$, energy discontinuities/coexistences in the actual small- $\mu$ systems whenever they are exhibited in the corresponding mean-field theory.

Let us start with some hypotheses on the energetics corresponding to the set $\mathcal{M}_{\beta}$ of minimizers for $\Phi_{\beta}(m)$ :

Definition 5.4. Regular Energy Hypothesis: Let $\Phi_{\beta}(m)$ denote a mean-field free energy function and $\Xi_{\eta}(\beta)$ denote the set of magnetizations such that $\Phi_{\beta}(m)$ is less than $F_{\mathrm{MF}}(\beta)+\eta$. Then the mean-field theory is said to satisfy the Regular Energy Hypothesis iffor all (sufficiently small) $\eta$ there is a $\delta(\eta)$ with $\delta(\eta) \rightarrow 0$ as $\eta \rightarrow 0$ and an $R$ - which does not depend on $\eta$ such that $\Xi_{\eta}(\beta)$ may be expressed as the union of $R$ separated sets

$$
\Xi_{\eta}=\bigcup_{r=1}^{R} \Xi_{\eta}^{(r)}
$$

with the properties: 
(i) Each $m \in \Xi_{\eta}^{(r)}$ has energy $\left(\propto m^{2}\right)$ that is within $\delta$ of some fixed $\mathcal{E}_{r}$ and

(ii) The sets $\Xi_{\eta}^{(r)}$ are decreasing (so the limiting set is either empty or of constant energy $\mathcal{E}_{r}$ ).

Remark 6. It is noted that the number, $R$, of such sets is allowed to shrink with $\eta$ (this is already within the technical leeway of the definition) but not allowed to grow - and especially not without bound. Basically these sets should be thought of as neighborhoods of certain sets of constant energy that minimize the free energy function at or near temperature parameter equal to $\beta$. Further, it is underscored that the values $\mathcal{E}_{r}$ need not themselves be separated, just the magnetizations within the sets $\Xi_{\eta}^{(r)}$ that these energies represent. Finally, concurrent with the Generic First Order Scenario, it seems that in all practical circumstances, the Regular Energy Hypothesis holds. However, in contrast to the former, it may well be possible to cook up a model where these hypotheses are violated.

Certain Mild Restrictions. Here we shall perform exercises on two scales: the local, $\ell_{0}$ which is small compared with $\mu^{-1}$ and the quasilocal, $\ell_{1}$ which will be large compared with $\mu^{-1}$. It will be convenient (not strictly necessary) to assume that $\ell_{1}$ is a multiple of $\ell_{0}$ and, even more so, that the lattice size $L$ is a power of two multiple of both $\ell_{0}$ and $\ell_{1}$. Moreover, in contrast to the magnetic results where no specific details were required concerning how $\mu \ell_{0} \rightarrow 0$, here some mild constraints will come into play; in particular, it will be necessary to ensure that $\ell_{1}$ does not go into infinity too fast relative to $\ell_{0}$. The restriction is indeed mild and is easily satisfied if $\ell_{1}$ is any superlinear power and $\ell_{0}$ any sublinear power of $\mu^{-1}$. In the forthcoming, often without specifics, all of the above will be referred to as the Mild Restrictions.

Definition 5.5. Consider a spin-system described by Eqs. (2.1)-(2.2) and suppose that the corresponding mean-field theory satisfies the Regular Energy Hypothesis. Let $\ell_{0}$ and $\ell_{1}$ be two length-scales satisfying, if appropriate, the Mild Restrictions. Let $\mathfrak{m}_{\ell_{0}}$ denote, as previously, the block average magnetization in $\Lambda_{\ell_{0}}$. For a of the form (integer vector $) \times \ell_{0}$, let $\Lambda_{\ell_{0}}(\underline{a})$ denote the translation of $\Lambda_{\ell_{0}}$ and $\mathfrak{m}_{\ell_{0}}(\underline{a})$ the block magnetization in $\Lambda_{\ell_{0}}(\underline{a})$. Let $\theta>0$ and $\eta$, etc. denote previous meanings. The block $\Lambda_{\ell_{1}}$ is said to satisfy the Thouroughgood condition of type $r$ (which is actually the $(\theta, \eta ; r)$-Thouroughgood condition) if

(1) For all a such that $\Lambda_{\ell_{0}}(\underline{a}) \subset \Lambda_{\ell_{1}}, \mathfrak{m}_{\ell_{0}}(\underline{a}) \in \Xi_{\eta}^{(r)}$.

(2) The Thouroughgood block energy defined by

$$
\mathcal{E}_{\ell_{1}}=-\frac{1}{2} \beta \frac{1}{\left|\Lambda_{\ell_{1}}\right|} \sum_{i, j \in \Lambda_{\ell_{1}}} J_{i, j}\left(\mathbf{s}_{i} \cdot \mathbf{s}_{j}\right)
$$

satisfies $\left|\mathcal{E}_{r}\right|-\left|\mathcal{E}_{\ell_{1}}\right|<\theta$.

It noted that if $\Phi_{\beta}(m)$ is minimized by isolated points (and $\eta$ is sufficiently small while $\theta$ is not too small) then condition (2) is trivially satisfied by condition (1). Condition (2) becomes interesting when there is a continuum of minimizing magnetizations which are all of the same "length".

Proposition 5.6. Consider an interaction of the type described in Eqs. (2.1)-(2.2) and suppose that the corresponding $\Phi_{\beta}$ satisfies the Regular Energy Hypothesis. Then, for 
any (small) $\eta$ there is a $\theta$ with $\theta(\eta)$ tending to zero such that as for all $\mu^{-1}$ and $\ell_{0}$, $\ell_{1}$ sufficiently large satisfying certain Mild Restrictions, the spins in $\Lambda_{\ell_{1}}$ satisfy the $(\theta, 3 \eta ; r)$-Thouroughgood condition for at least one value of $r$ with probability tending to one.

Proof. Let us begin by ruling out the possibility of not satisfying criterion (1). This can come about in two ways: First, one of the subblocks $\Lambda_{\ell_{0}}(\underline{a})$ can satisfy the analogue of $\mathbb{B}_{\left[\ell_{0}\right], 3 \eta}$ (meaning that $\mathfrak{m}_{\ell_{0}} \notin \Xi_{3 \eta}(\beta)$ ). Here we may use Eq. (5.14) and over-count the location. The Mild Condition will force

$$
\left[\frac{\ell_{1}}{\ell_{0}}\right]^{d} K(\eta) e^{-\eta \ell_{0}^{d}} \rightarrow 0
$$

as $\mu \rightarrow 0$. Next there is the possibility that all subblocks are in good shape magnetically but there are specimens that have differing $r$-phenotype. This, by necessity, will result in a mismatched neighboring pair. Such a possibility can be demonstrated as unlikely by a standard chessboard estimate: Suppose that there are two (particular) neighboring blocks with respective block magnetizations in $\Xi_{3 \eta}^{(r)}$ and $\Xi_{3 \eta}^{(s)}$ with $s \neq r$. Reflecting this event till the torus is covered, it is found that in one direction (along the direction of the pair) there is a dashed pattern and this dash gets extended into the other $(d-1)$-directions - stripes, plates, etc. Let us denote the constrained partition function on the torus $\mathbb{T}_{L}$ by $Z_{L, \beta}(r, s)$. For some fixed $\Delta$-scale, the entropy of $Z_{L, \beta}(r, s)$ can be estimated along the lines of Eqs. (5.11)-(5.13) resulting in a factor of

$$
\left[\left[\tilde{G}_{r} \tilde{G}_{S}\right]^{\ell_{0}^{-d}} e^{\frac{1}{2}\left[S\left(m_{r}\right)+S\left(m_{s}\right)+\epsilon_{1}\right]}\right]^{\left|\mathbb{T}_{L}\right|},
$$

where $S\left(m_{r}\right), S\left(m_{s}\right)$ are mean-field entropies representative of the sets $\Xi_{3 \eta}^{r}$ and $\Xi_{3 \eta}^{s}-$ note that the entropy cannot vary much in these sets - the $\tilde{G}$ (which depend on $\eta$ ) are the appropriate analogs of the $K(\eta)$ that has appeared before and $\epsilon_{1}$ is a tolerable error - vanishes with $\eta$. Let us turn to the energetics of $Z_{L, \beta}(r, s)$.

Let $\underline{\mathbf{r}}$ and $\underline{\mathbf{s}}$ denote the sublattice of blocks covered by the two types of events. Following Eqs. (5.7)-(5.10) we may write

$$
\sum_{i, j} J_{i, j}\left(\mathbf{s}_{i} \cdot \mathbf{s}_{j}\right) \approx\left|\Lambda_{\ell_{0}}\right|^{2} \sum_{p, q} J_{p, q}\left(m_{p} \cdot m_{q}\right)=\left|\Lambda_{\ell_{0}}\right|^{2} \sum_{p}\left(m_{p} \cdot \sum_{q} J_{p, q} m_{q}\right)
$$

with formal acknowledgment of the small debt from the first step to be made later on. Let us look at the inner summand in the last term - with fixed $p$ - and suppose that $p \in \underline{\mathbb{}}$. The terms where $q \in \underline{\mathbb{r}}$ may be replaced, as an upper bound, by $\frac{2}{\beta}\left(\left|\mathcal{E}_{r}\right|+\delta\right)-$ essentially $m_{p}^{2}$. Adding and subtracting a "favorable" term, namely $m_{p}$, for $q \in \underline{\mathbf{s}}$ we arrive at

$$
\left(m_{p} \cdot \sum_{q} J_{p, q} m_{q}\right) \leq \frac{1}{\left|\Lambda_{\ell_{0}}\right|} \frac{2}{\beta}\left(\left|\mathcal{E}_{r}\right|+\delta\right)+\left(m_{p} \cdot \sum_{q \in \underline{\mathbb{S}}} J_{p, q}\left[m_{q}-m_{p}\right]\right) .
$$

When this gets (multiplied by $\left|\Lambda_{\ell_{0}}\right|^{2}$ and) summed over $p \in \underline{\mathbb{r}}$ the $\mathcal{E}_{r}$-type term will be half what is expected for an energetic contribution because $\underline{\mathbf{r}}$ is half of the lattice. 
Performing the same routine if $p \in \underline{\mathbf{s}}$ and putting additional required factors of $\beta$ and $\frac{1}{2}$ yields

$$
\begin{aligned}
\frac{1}{2} \beta \sum_{i, j} J_{i, j}\left(\mathbf{s}_{i} \cdot \mathbf{s}_{j}\right) \leq & {\left[\frac{1}{2}\left|\mathcal{E}_{r}\right|+\frac{1}{2}\left|\mathcal{E}_{S}\right|+\epsilon_{2}\right]\left|\Lambda_{L}\right| } \\
& -\frac{1}{2} \beta\left|\Lambda_{\ell_{0}}\right|^{2} \sum_{\substack{p \in \mathbb{\mathbf { r }} \\
q \in \underline{\mathbf{S}}}} J_{p, q}\left(m_{p}-m_{q}\right)^{2}
\end{aligned}
$$

with $\epsilon_{2}$ another tolerable error term which also accounts for the neglect in Eq. (5.18). The final term cannot be diminished. The minimal squared distance between any pair magnetizations with one in $\Xi_{3 \eta}^{(r)}$ and the other in $\Xi_{3 \eta}^{(s)}$ will be denoted by $\frac{2}{\beta} \mathcal{V}_{r, s}$ with $\mathcal{V}_{r, s}$ strictly positive by the Regular Energy Hypothesis. It is noted that as $\mu \rightarrow 0$, then $\left|\Lambda_{L}\right|^{-1}\left|\Lambda_{\ell_{0}}\right|^{2}$ times the sum of the $J_{p, q}$ tends to $\frac{1}{4}-$ let us denote a (uniform) lower bound, valid for all $\mu$ sufficiently small, by $\kappa$; we have arrived at

$$
Z_{L, \beta}(r, s)^{1 /\left|\mathbb{T}_{L}\right|} \leq\left[\left[\tilde{G}_{r}\right]^{\ell_{0}^{-d}} e^{\frac{1}{2}\left(\left|\mathcal{E}_{r}\right|+S\left(m_{r}\right)\right)}\left[\tilde{G}_{s}\right]^{\ell_{0}^{-d}} e^{\frac{1}{2}\left(\left|\mathcal{E}_{s}\right|+S\left(m_{s}\right)\right)} e^{\epsilon_{3}}\right] \times e^{-\ell_{0}^{d} \kappa \mathcal{V}_{r, s}}
$$

with all previously discussed errors amalgamated into the final $\epsilon_{3}$. Aside from terms that are close to unity, the term above in the large square bracket is identified as a negative exponent of the mean-field free energy which, e.g. according to Eq. (5.6), is canceled by the denominator in the chessboard estimate.

Thus the probability of a particular mismatched neighboring pair of (otherwise decent) subblock magnetizations is bounded by a quantity that is exponentially small with rate $\propto\left|\Lambda_{\ell_{0}}\right|$. Accounting for all possible locations and all possible types of mismatches multiplies this by a constant (which depends on $R$ and $d$ ) times $\left|\Lambda_{\ell_{1}}\right| /\left|\Lambda_{\ell_{0}}\right|$ so, overall, is actually more heavily suppressed than the situation where one of the blocks had a "bad magnetization". In any case, it may be declared that criterion (1) is satisfied with high probability.

Let us turn to criterion (2). As may already be obvious, a central reason for the stipulation $\ell_{0} \ll \mu^{-1} \ll \ell_{1}$ is that the total energy of most spins in $\Lambda_{\ell_{1}}$ is accounted for by the pairings with other spins in $\Lambda_{\ell_{1}}$. Indeed, this reasoning only breaks down for sites that are a distance of order $\mu^{-1}$ from the edge; let us denote

$$
Q\left(\mu, \ell_{1}\right)=\sum_{\substack{i \in \Lambda_{\ell_{1}} \\ j \in \Lambda_{\ell_{1}}^{c}}} J_{i, j},
$$

then it is not hard to show, if $\mu \ell_{1} \rightarrow \infty$ with $\mu \rightarrow 0$, that $\left[\mu^{-1} \ell_{1}^{d-1}\right]^{-1} Q\left(\mu, \ell_{1}\right)$ tends to a definitive constant. So, letting $\mathbb{B}_{\left[\ell_{1}\right], r}^{(1) \backslash(2)}$ denote the event that the spins on $\Lambda_{\ell_{1}}$ satisfy, for energy $\mathcal{E}_{r}$, the Thouroughgood criterion (1) but not criterion (2), let us perform another chessboard estimate. Let us use $Z_{L, \beta}\left(\mathbb{B}_{\left[\ell_{1}\right], r}^{(1) \backslash(2)}\right)$ to denote the constrained partition function; it is clear that the entropy is, more or less, $\left|\mathbb{T}_{L}\right| S\left(m_{r}\right)$ but the energetic contribution is no more than $\left[\left|\mathcal{E}_{r}\right|-\theta+\left|\Lambda_{\ell_{1}}\right|^{-1} Q\left(\mu, \ell_{1}\right) \cdot \frac{1}{2} \beta \omega^{2}+\epsilon_{4}\right]\left|\mathbb{T}_{L}\right|-$ all terms understood to be appearing in the exponent - with the $\omega^{2}$ term representing a bound on the largest conceivable energetic contribution to the configuration coming from interactions between spins in differing blocks. Canceling, as before, the mean-field free energy term from 
numerator and denominator, taking the appropriate power, namely $\ell_{1}{ }^{d} /\left|\mathbb{T}_{L}\right|$, we arrive at the estimate for $\alpha_{\beta, \mathbb{T}_{L}}\left(\mathbb{B}_{\left[\ell_{1}\right], r}^{(1) \backslash(2)}\right)$ of $e^{-\left[\theta-\epsilon_{5}\right] \ell_{1}^{d}}$ for yet another $\epsilon_{j}$ and the proposition is proved.

Remark 7. In essence, the above proposition already tells us that in case $R=1$, the actual system will have energy close to the mean-field value. If the mean-field theory is sufficiently regular (and let us not pause to axiomatize the concept) it would seem that except at points of a discontinuity, the large $\mu^{-1}$ system will follow the mean-field theory. However, in isolation, this result is not all that illuminating - especially if one considers that so far, there has been no stipulation that we are not in $d=1$. Indeed, let us assume that the mean-field theory has a discontinuous transition in the energy at some $\beta_{t}$ and that the actual system is at some nearby $\beta$. Then, in order to ensure that the minimizers on the other side of the transition are excluded from $\Xi_{3 \eta}$, smaller and smaller $\eta$ 's have to be chosen as $\beta \rightarrow \beta_{t}$. This in turn necessitates increasingly larger values of $\mu^{-1}$ in order to bring the results of Proposition 5.6 into play. Thus a non-uniform type of convergence will transpire in the vicinity of a mean-field transition temperature - a result which, after a moments thought, one always anticipates, even in $d=1$. Our next result, which definitively requires $d \geq 2$, shows that, under reasonable hypotheses, systems with a large enough range parameter are (uniformly) close to some energy corresponding to a near-minimizer of the mean-field theory. This, of course, allows us to keep $\eta$ fixed at the "expence" of multiple possibilities for the energy.

Proposition 5.7. Consider a spin-system on $\mathbb{Z}^{d}$ with $d \geq 2$ that is described by the interaction in Eqs. (2.1)-(2.2) and suppose that at temperature parameter $\beta$, the associated mean-field theory satisfies the Regular Energy Hypothesis. Then, for all $\mu$ sufficiently small, there is an $\eta$ and $a \tilde{\delta}(\eta)$ and a set of infinite-volume Gibbs states emerging from the $\alpha_{\beta, \mathbb{T}_{L}}$ such that with probability one, the energy density in any configuration is within $\tilde{\delta}$ of a value $\mathcal{E}_{r}, r=1,2, \ldots R$ associated with $\Xi_{3 \eta}^{(r)}$. Moreover, if the hypotheses hold with a uniform bound on the separations between the various sets $\Xi_{3 \eta}^{(r)}$ while $\beta$ ranges over a compact set, then with $\eta$ and $\tilde{\delta}$ fixed (and $\eta$ sufficiently small) the result holds uniformly for all $\mu$ below some minimal value.

Proof. For a system of the type described above, let $\eta>0$ and let $\theta(\eta)$ denote the quantity described in Proposition 5.6. If $R=1$, the argument is somewhat simpler but in any case, let us employ an argument appropriate to $R \geq 2$. It is observed that if two distinct "blocks" - translates of $\Lambda_{\ell_{1}}$ by lattice vectors with components (integer) $\times \ell_{1}$ - are of different Thouroughgood energy type, then these blocks are separated by a closed $*$-connected contour consisting of non-Thouroughgood blocks or Thouroughgood blocks that interface with a Thouroughgood block of a different energy type. The former sort of contour element was, manifestly, estimated in the previous proposition. As we shall see, so has most of what is needed for the latter. Indeed, supposing that the two energy types are $r$ and $s$, let us neglect all aspects of the interface event save for the fact that there is a row of $\ell_{1} / \ell_{0}$ boxes which are translates of $\Lambda_{\ell_{0}}$ that have their magnetization in $\Xi_{3 \eta}^{(r)}$ and this row faces a similar opposing row with magnetizations in $\Xi_{3 \eta}^{(s)}$. When all this gets reflected to cover the torus, the constrained partition function is exactly $Z_{L, \beta}(r, s)$. This time, our estimate will be 


$$
\left[Z_{L, \beta}^{-1} Z_{L, \beta}(r, s)\right]^{\frac{2 \ell_{0} \ell_{1}^{d-1}}{\left|\mathbb{T}_{L}\right|}}
$$

which is a tremendous - albeit not unexpected - enhancement of the previous run through.

Thus it is claimed that with high probability, on $\mathbb{T}_{L}$ most blocks are situated exterior to contours. In particular, let us denote by $\vartheta$ the estimate for contour elements - the probability that a given block belongs to a contour of length $k$ is bounded above by a constant times $\vartheta^{k}$. Then, it is not difficult to show that if $H$ is any appreciable number (perhaps not too large but certainly in excess of unity) the probability that a fraction larger than $\vartheta H$ of all blocks reside inside or on contours tends to zero exponentially at an estimated rate that is a (sublinear) power of the volume. Since results of this sort are well known and the subject of many works in specific systems, let us proceed with a terse, highly non-optimal derivation.

Let us suppose that we desire $H \vartheta\left[L \ell_{1}^{-1}\right]^{d}$ or more non-exterior boxes. We shall say that contours which have between $T$ and $2 T$ elements have done their share if they produce at least $\frac{1}{T} H \vartheta\left[L \ell_{1}^{-1}\right]^{d}$ such boxes. Starting at $T=1$ and proceeding along powers of 2 , it is clear that if none of the groupings have done their share, the event has failed. Let us start (and end) with all $T$ 's that satisfy $T>T_{0}=\left[L \ell_{1}^{-1}\right]^{a_{0}}$, where $a_{0}>0$ is to be determined below. There simply are no such contours with probability greater than $1-b_{1}\left[L \ell_{1}^{-1}\right]^{d} \vartheta^{T_{0}}$, where $b_{1}$ is a constant of order unity. So it is fairly safe to assume that none of these have done their share. For $T$ 's that are smaller, let us go to a block lattice with cell size of e.g. $8 T \ell_{1}$, and focusing on a sublattice of $2 T \ell_{1}$, ask if any "site" in this part of the cell belongs to a contour of size between $T$ and $2 T$. If yes, we surrender the whole cell and relax the criterion of "share" accordingly. Still this requires $T^{-a_{1}} H_{1} \vartheta\left[L \ell_{1}^{-1}\right]^{d}$ successes out of a total of $c_{1}\left[L T^{-1}\right]^{d}$ trials with a probability bounded by $T^{a_{2}} \mathrm{H}_{2} \vartheta^{T}$ for each success; the latter is estimated by chessboard methods. In the above, $a$ 's, $H$ 's etc. are of order unity with $H_{1}$ numerically large if $H$ is large. The upshot, for a fair share at scales between $T$ and $2 T$ is an upper estimate of the form

$$
\left(\frac{T^{a_{3}} \vartheta^{T-1}}{H_{3}}\right)^{c_{2} T^{-a_{1}} \vartheta H_{1} L^{d}}
$$

with all constants of order unity and both $H_{1}$ and $H_{3}$ large if $H$ is large. Clearly the above gets out of hand if we let $T$ get too large but we shall cut off when the above approximately matches our preliminary estimate - which determines the value $T_{0}$. For all other $T$ except, perhaps, for the very first few, this will be small due to the $\vartheta$ term and the cases $T \sim 1$ can rely on large $H$ or (which essentially amounts to the same thing) can be done by hand.

With the vast majority of blocks in the exterior of contours, it is indeed the case that the energy content is close to $\mathcal{E}_{r}$ for some $r$ - here another estimate using $Q\left(\mu, \ell_{1}\right)$ is employed. Finally it is noted that all estimates in this and the previous proposition stem from the initial estimate in the first few lines of Proposition 5.6 for which uniformity was established in Corollary 5.2. All subsequent rates, bounds, etc. depend trivially on the separations between the $\Xi_{3 \eta}^{(r)}$, s - which have been deemed to have a minimal value $-\eta, \theta$ and various other parameters can be determined by a worst case scenario on a bounded interval of $\beta$ 's.

Corollary 5.8. Consider a spin-system satisfying the hypotheses of Proposition 5.7. Then the conclusion of this proposition holds, perhaps with a slight adjustment of $\tilde{\delta}$, in every shift invariant ergodic measure which is a Gibbs state for the interaction. 
Proof. The desired result is, almost, an immediate application of Theorem 2.5 and Corollary 2.6 in [5] save for the fact that here (and in various other places throughout this work) the relevant "good" events do not quite satisfy their hypotheses in cases where there are multiple types of goodness. However this can be circumvented by the construction of a superblock: Let us introduce one more length scale, $\ell_{2}$ with $\ell_{2} \gg \ell_{1}$. In contrast to the previous $\ell_{j}$, this length will not be tied to $\mu$ or any other parameters. On the contrary, it is envisioned that $\ell_{2} \rightarrow \infty$ with all other quantities fixed. However it will be assumed, for connivance, that $\ell_{1}$ and $L$ are related to $\ell_{2}$ by powers of two.

Consider the superblock event, defined on $\Lambda_{\ell_{2}}$ that all but a fraction $\vartheta^{\star}$ of the tiling $\Lambda_{\ell_{1}}$-sized subblocks satisfy the Thouroughgood condition for the same value of $r$. If this event is denoted by $g^{\star}\left(\vartheta^{\star}\right)$, arguments along the lines of those in Proposition 5.7 show that

$$
\alpha_{\beta, \mathbb{T}_{L}}\left(\mathcal{I}^{\star}\right) \leq e^{-\mathcal{K}(\vartheta \star)\left[\frac{\ell_{2}}{\ell_{1}}\right]^{v}}
$$

for some positive power $v$ and $\mathcal{K}$ positive once $\vartheta^{\star}$ is an appreciable multiple of the estimate in the final line of Proposition 5.6.

Now let $\alpha_{\beta}$ denote any ergodic Gibbs state corresponding to the specified Hamiltonian and suppose that the energy density, $\mathcal{E}$, of $\alpha_{\beta}$ is not within the appropriate $\delta$ of any $\mathcal{E}_{r}$. Then, with high probability, the $\alpha_{\beta}$-energy per site of a sufficiently large block (i.e. $\Lambda_{\ell_{2}}$ with $\ell_{2}$ sufficiently large) is also outside of the anticipated range. In light of the estimate in Eq. (5.23) this is not permitted by the above mentioned theorem in [5].

Corollary 5.9. For spin-systems satisfying the above hypotheses, there is an $\eta^{\prime} \gtrsim 3 \eta$ such that in any shift invariant ergodic Gibbs state, in almost every configuration the magnetization is in $\operatorname{Conv}\left(\Xi_{\eta^{\prime}}^{(\mathrm{r})}\right)$ for some r.

Proof. The result follows immediately from the preceding (and continuity of $\Phi_{\beta}$ ). Indeed, in this case, the superblock construction can proceed without the benefit of the intermediate scale.

Remark 8 . For some systems, e.g. when $\mathcal{M}_{\beta}$ consists only of isolated points, the above is in essence the final result. But in others, e.g. the $O(n)$-systems, this corollary basically provides no information.

It is not difficult to imagine that, with the insertion of some further energy hypotheses, we would be in position to directly establish discontinuous transitions in the energy density for "real" systems in $d \geq 2$ whenever such transitions occur in the mean-field theory. However, the necessary hypotheses turn out to be slightly nebulous in appearance. Hence we will follow the alternate route of tracking the magnetizations - which in any case are closely tied to the energies - and the results of this subsection will be utilized in a supporting rôle.

\subsection{Proofs of main results.}

Proof of Main Theorem. With what has so far been established, we are in prime position to apply the classic result of Kotecký and Shlosman, which provides a sufficient condition for the occurrence of a $1^{\text {st }}$ order transition. For completeness, let us summarize the hypotheses of [23] Theorem 4 (which have been abbreviated by limiting attention to circumstances where the relevant numerical parameters are small quantities). 
Consider a spin-system on $\mathbb{Z}^{d}, d \geq 2$ belonging to a certain class which includes that defined by $\mathcal{H}$ in Eq. (2.1)-(2.2) and suppose there are events $A_{I}$ and $A_{I I}$ defined on the block $\Lambda_{\ell_{0}}$ and an interval of inverse temperature $\left[\beta_{I}, \beta_{I I}\right]$ such that for certain small numbers $a_{1}, a_{2}, b_{1}$ and $b_{2}$ and some $L_{0}$, the following holds for infinitely many $L$ 's that are larger than $L_{0}$ :

(i) For all $\beta \in\left[\beta_{I}, \beta_{I I}\right], \alpha_{\beta, \mathbb{T}_{L}}\left(\left[A_{I} \cup A_{I I}\right]^{c}\right)<b_{1}$.

(ii) The $\lim _{\beta \rightarrow \beta_{I}} \alpha_{\beta, \mathbb{T}_{L}}\left(A_{I}\right) \geq 1-a_{1}$.

(iii) The $\lim _{\beta \rightarrow \beta_{I I}} \alpha_{\beta, \mathbb{T}_{L}}\left(A_{I I}\right) \geq 1-a_{2}$.

(iv) For all $\beta \in\left[\beta_{I}, \beta_{I I}\right]$, if $\tau_{j}\left(A_{I}\right)$ denotes the event $A_{I}$ translated to the lattice site $j$, then for all $j, \alpha_{\beta, \mathbb{T}_{L}}\left(\tau_{j}\left(A_{I}\right) \cap A_{I I}\right)<b_{2}$.

Then there is a $\beta_{t} \in\left(\beta_{I}, \beta_{I I}\right)$ such that at $\beta=\beta_{t}$, there are at least two coexisting Gibbs states corresponding to $\mathcal{H}$, denoted by $\alpha_{\beta_{t}}^{I}$ and $\alpha_{\beta_{t}}^{I I}$ that are distinguished by

$$
\alpha_{\beta_{t}}^{I}\left(A_{I}\right) \geq 1-c ; \alpha_{\beta_{t}}^{I I}\left(A_{I I}\right) \geq 1-c,
$$

where $c$ is small if the $a$ 's and $b$ 's are small.

It is clear that for a Generic First Order Scenario, we may utilize the events $A_{I}=\left\{\mathfrak{m}_{\ell_{0}} \in \mathbb{M}_{I}\right\}$ and similarly for $A_{I I}$. Using the hypothesis of the scenario and Lemma 5.1, items (i) - (iii) are satisfied; let us turn to (iv).

For the latter, we shall adapt some previous notation: If $j \in \mathbb{Z}^{d}$, let $\mathfrak{m}_{\ell_{0}}(j)$ denote the average magnetization in $\tau_{j}\left(\Lambda_{\ell_{0}}\right)$ - thus $\tau_{j}\left(A_{I I}\right)$ is the event $\left\{\mathfrak{m}_{\ell_{0}}(j) \in \mathbb{M}_{I I}\right\}$. Now let us define a site $i$ to be good if $\mathfrak{m}_{\ell_{0}}(i) \in \mathbb{M}_{I} \cup \mathbb{M}_{I I}$ and otherwise bad. It is first noted that $A_{I} \cap A_{I I}=\varnothing$ (and similarly for the translations) since by hypothesis, $\mathbb{M}_{I}$ and $\mathbb{M}_{I I}$ are separated. Thus there are two types of good sites. Now suppose that the origin is of type I and $j$ is of type II, i.e. the event $A_{I} \cap \tau_{j}\left(A_{I I}\right)$; let us consider the connected component of type I good sites of the origin. We will use the convention that a boundary site is outside the cluster with a neighbor in the cluster. A boundary site could, ostensibly, be a bad site or a site of type II. However, we use the condition $\ell_{0} \gg 1$ and the obvious fact that for any lattice vector $\hat{e}_{i}$,

$$
\left|\mathfrak{m}_{\ell_{0}}\left(j+\hat{e}_{i}\right)-\mathfrak{m}_{\ell_{0}}(j)\right| \leq \frac{\omega}{\ell_{0}^{d-1}},
$$

where $\omega$ is as big as a spin can get. As a consequence, since $\mathbb{M}_{I}$ and $\mathbb{M}_{I I}$ are separated, if $\ell_{0}$ is large enough, the boundary of any region of type I sites must actually be bad sites. We thus have certain contours and contour events - which will typically be denoted by $\gamma$; these are, technically, $*-$ connected contours, that is to say neighbors and next-nearest neighbors are considered connected. It is further remarked that there are actually two types of contours possible depending on "who is separated from whom" plus the possibility of a contour that winds the torus (an SSWC-contour) all of which can be accounted for by doubling the estimate obtained by an a priori infinite sum over contours. Let us focus on the more pertinent issues:

Foremost, the events that the individual contour elements (the sites of $\gamma$ ) represent are actually defined on the larger scale $\ell_{0}$ and, even using reflection positivity methods, it is not possible to obtain a tractable Peierls-type estimate without a bit of course-graining. Thus, let us formally consider the lattice $\mathbb{T}_{L / \ell_{0}}$ whose "sites" consist of the disjoint blocks that are appropriate translates of $\Lambda_{\ell_{0}}$. If $\gamma$ denotes a microscopic $*$-connected contour (or any path) we may associate a cluster, $\Gamma=\mathbb{Q}(\gamma)$, on $\mathbb{T}_{L / \ell_{0}}$ representing the blocks of scale $\ell_{0}$ that were visited by $\gamma$. Notice that $\Gamma$ may itself be only vaguely 
contour-like but, at any rate, it is a $*$-connected object. Now if $J \in \mathbb{T}_{L / \ell_{0}}$ denotes a (block) site, the probability that $J$ belongs to a course grained contour element is exactly the probability that some site in the block is bad. This, in term, may be bounded by the volume of the block times the estimate on the right side of Eq. (5.14). Let us use $\varepsilon_{C}=\varepsilon_{C}\left(\ell_{0}, \mu, \eta\right)$ to denote this small quantity.

A secondary (minor) obstruction occurs for a block contour event associated with a $\Gamma$. Indeed we cannot use chessboard methods on each block-element since the relevant events in $*$-neighboring blocks may be entangled. However, disjoint sublattices on $\mathbb{T}_{L / \ell_{0}}$ may be considered such that the blocks on each sublattice are devoid of $*$-neighbors in their own sublattice. In $d=2$ there are four such sublattices, in general it is $2^{d}$. Thus, finally, for each (admissible) cluster $\Gamma$, let $|\Gamma|$ denote the maximum of the number of blocks of $\Gamma$ which reside on the various sublattices.

The argument can now be finished along standard lines. The block contour event where the cluster is of size $N$ must be within the distance of the order $N$ of the block containing $i$ or the block at the origin. The number of such clusters is therefore bounded by $A(d) N^{a(d)} e^{\kappa(d) N}$ with all constants finite and the necessary "double counting" folded into these constants. Therefore, defining

$$
\Gamma_{N}=\{\exists \gamma \text { of bad sites separating } 0 \text { from } i \text { with }|\mathbb{Q}(\gamma)|=N\}
$$

we have

$$
\alpha_{\beta, \mathbb{T}_{L}}\left(\Gamma_{N}\right) \leq A N^{a} e^{\kappa N} \varepsilon_{C}^{N}
$$

Summing from $N=1$ the result is small if $\varepsilon_{C}$ is small and, under the hypotheses concerning $\ell_{0}, \mu$, etc. condition (iv) has been verified.

Proof of Proposition 3.1. This is, in essence, the $2^{\text {nd }}$ corollary to Proposition 5.7 (Corollary 5.9). First, since the sets $M^{(j)}$ are convex and separated then small neighborhoods of these sets - large enough to contain the appropriate $\Xi_{\eta^{\prime}}^{(j)}$ - are convex and separated. Thus the magnetization is always in one of these neighborhoods and there is at least one Gibbs state of the specified form. But now, due to the invariance of the interaction, if there is a Gibbs state associated with one of the $M^{(j)}$, then there is a Gibbs state for all the others as well.

Proof of Theorem 3.2. Under the hypotheses off a Generic First Order Scenario, the result is established by the Main Theorem (which proves a transition between $M_{I}$ and $M_{I I}$ - like states) and Proposition 3.1 which establishes the nature of the Gibbs states. Alternatively, with the hypothesis of an energy gap between $M_{I}$ and $M_{I I}$, a Generic First Order Scenario is readily established. Let us start by finding a $\delta m$ which is small compared to all separations between the various $M_{J}^{(j)}$; explicitly that the $\delta m$-neighborhoods of these sets are still separated. Next, let us define an $\underline{\kappa}$ which is small enough so that $\Xi_{\kappa}\left(\beta_{t}^{\mathrm{MF}}\right)$ is contained in the union of these neighborhoods. Notice that there is an unambiguous $\Xi_{\kappa}^{I}\left(\beta_{t}^{\mathrm{MF}}\right)$, similarly for $I I$ and also for the various offshoots from the $M_{J}^{j}$. The quantity $\underline{\kappa}$ will define both the temperature scale and, for all intents and purposes the $($ three $\times) \eta$. Let $\left[\beta_{I}, \beta_{I I}\right]$ be the symmetric interval about $\beta_{t}^{\mathrm{MF}}$ that has, to be definitive,

$$
\underline{\kappa}=\omega^{2}\left(\beta_{I I}-\beta_{I}\right)
$$


(and notice that this necessarily implies that $\omega^{2}\left(\beta_{I I}-\beta_{I}\right) \ll \beta_{t}^{\mathrm{MF}}\left(m_{I}-m_{I I}\right)^{2}$ for any $m_{J} \in M_{J}$ ). Finally $3 \eta$ will satisfy

$$
3 \eta<\frac{1}{2} \frac{\beta_{I I}-\beta_{I}}{\beta_{t}^{\mathrm{MF}}} \Delta_{\mathcal{E}}
$$

so, except for the possibility of some terrible anomaly in the sizes of the $m$ 's in $\mathcal{M}_{\beta_{t}^{\mathrm{MF}}}, \eta$ and $\underline{\kappa}$ are comparable. Now define

$$
\mathbb{M}_{I}=\bigcup_{\beta \in\left[\beta_{I}, \beta_{I I}\right]} \Xi_{3 \eta}^{I}(\beta)
$$

and similarly for $\mathbb{M}_{I I}$. Let us demonstrate that $\mathbb{M}_{I} \cup \mathbb{M}_{I I}$ is contained in $\Xi_{\kappa}-$ here repeated use will be made of the identity $\Phi_{\beta^{\prime}}(m)=\Phi_{\beta^{\prime \prime}}(m)-\frac{1}{2}\left(\beta^{\prime}-\beta^{\prime \prime}\right) m^{2}$. Suppose that $m \notin \Xi_{\underline{\kappa}}\left(\beta_{t}^{\mathrm{MF}}\right)$. Then, for $\beta \in\left[\beta_{I}, \beta_{I I}\right]$,

$$
\begin{aligned}
\Phi_{\beta}(m) & \geq F_{\mathrm{MF}}\left(\beta_{t}^{\mathrm{MF}}\right)+\underline{\kappa}+\frac{1}{2}\left(\beta_{t}^{\mathrm{MF}}-\beta\right) m^{2} \\
& \geq F_{\mathrm{MF}}(\beta)-\frac{1}{2}\left|\beta_{t}^{\mathrm{MF}}-\beta\right| \omega^{2}+\underline{\kappa}-\frac{1}{2}\left|\beta_{t}^{\mathrm{MF}}-\beta\right| m^{2} \\
& \geq F_{\mathrm{MF}}(\beta)-\frac{1}{2}\left(\beta_{I I}-\beta_{I}\right) \omega^{2}+\underline{\kappa}=F_{\mathrm{MF}}(\beta)+\frac{1}{2} \underline{\kappa},
\end{aligned}
$$

and since, certainly, $3 \eta<\frac{1}{4} \omega^{2}\left(\beta_{I I}-\beta_{I}\right)$, it is clear that $m \notin \Xi_{3 \eta}(\beta)$. Notice that this also implies that there is a separated $\mathbb{M}_{I}$ and $\mathbb{M}_{I I}$ which in turn consist of $k_{I}$ and, respectively, $k_{I I}$ subsets associated with the $M_{J}^{(j)}$.

Let us see that the hypotheses of the Scenario are satisfied. Item (i) is our starting premise. Items (iia) and (iic) have been constructed with the identification of $3 \eta$ with $\zeta$. Item (iid) is an obvious consequence of continuity. We are left with item (iib) which is to show that at $\beta=\beta_{I I}$, the set $\mathbb{M}_{I I}$ contains all the minimizers in the strong sense that $F_{\mathrm{MF}}\left(\beta_{I I}\right)$ falls below $\Phi_{\beta_{I I}}\left(m_{I}\right)-3 \eta$ for all $m_{I} \in \mathbb{M}_{I}$. And we will need the corresponding statement for $F_{\mathrm{MF}}\left(\beta_{I}\right)$. This follows from an argument similar to the above. Let $m_{I} \in \mathbb{M}_{I}$. Then for $m_{I I} \in M_{I I} \subset \mathbb{M}_{I I}$,

$$
\Phi_{\beta_{I I}}\left(m_{I}\right)-\Phi_{\beta_{I I}}\left(m_{I I}\right)=\Phi_{\beta_{t}^{\mathrm{MF}}}\left(m_{I}\right)-\Phi_{\beta_{t}^{\mathrm{MF}}}\left(m_{I I}\right)+\frac{1}{4}\left(\beta_{I I}-\beta_{I}\right)\left(m_{I I}^{2}-m_{I}^{2}\right) .
$$

Now $\Phi_{\beta_{t}^{\mathrm{MF}}}\left(m_{I I}\right)=F_{\mathrm{MF}}\left(\beta_{t}^{\mathrm{MF}}\right)$ and $\Phi_{\beta_{t}^{\mathrm{MF}}}\left(m_{I}\right)$ cannot be lower. Meanwhile, $\left(m_{I I}^{2}-\right.$ $\left.m_{I}^{2}\right) \geq\left[2 / \beta_{t}^{\mathrm{MF}}\right] \Delta_{\mathcal{E}}$; obviously all the minimizers are in $\mathbb{M}_{I I}$ and moreover, the gap is at least $3 \eta$. A similar argument holds at the other end of the interval and the proof of a first order transition is complete. The remainder of the statements follow from the first portion of the proof and/or are automatic.

Proof of Theorem 3.3. Without loss of generality the treatment shall be confined to the case where $m_{1}$ and $m_{2}$ are the preferred approximate magnetizations destined for coexistence. Armed with Lemma 5.1 and its corollary, most of the proof amounts to an exercise in linear algebra and analysis. First, by the Gramm-Schmidt procedure (using the inner product defined by the interaction in Eq. (2.1)) let us consider an orthonormal set of fields starting with $\hat{b}_{1}$ and $\hat{b}_{2}$ covering the span of $m_{1}$ and $m_{2}$ with, say, $\hat{b}_{1} \propto m_{1}$. The successive fields, $\hat{b}_{3}, \ldots \hat{b}_{k}$ are now orthogonal to $m_{1}$ and $m_{2}$, thus their addition to the 
Hamiltonian as described in the statement of this theorem hardly effects the free energy function in the vicinity of these points.

The strategy will be to first use these last $k-2$ fields to suppress the "unwanted" states and then employ $\hat{b}_{1}$ and $\hat{b}_{2}$ in tandem to enhance one of $\left\{m_{1}, m_{2}\right\}$ at the expense of the other. First let $\varsigma$ denote a small quantity and consider the $\varsigma$-neighborhoods of $m_{j}$ which will be denoted by $\mathcal{N}_{\zeta}\left(m_{j}\right)$ and which may be assumed to be disjoint from one another. Ultimately the applied external field, $b_{\lambda}^{1,2}$, will be small depending on $\varsigma$ and we need not search outside of $\cup_{j} \mathcal{N}_{S}\left(m_{j}\right)$ for a minimizer of the augmented free energy function. Indeed, letting $\vartheta$ denote the minimal surplus outside these regions:

$$
\inf \left\{\Phi_{\beta}(m) \mid m \in\left[\cup_{j} M_{j}^{\varsigma}\right]^{c}\right\}=F_{\mathrm{MF}}(\beta)+\vartheta,
$$

it is obviously sufficient that $\left\|b_{\lambda}^{1,2}\right\|_{2}$ stay bounded by a constant times $\vartheta$, e.g. $\vartheta / 4 \omega$ where, it is recalled, $\omega$ is the limiting size of the magnetization in all of $\Omega$. Let us start with the construction of the "suppressor fields"; for convenience we shall work with the fields $\tilde{b}_{j} \propto \hat{b}_{j}$ that satisfy $\left(\tilde{b}_{j} \cdot m_{j}\right)=1$. Let $c>1$ denote a constant and let us define coefficients $\gamma_{3}, \ldots \gamma_{k}, \gamma_{j} \geq 1$ and, say, $\gamma_{3}=1$ such that

$$
\gamma_{j}=\gamma_{j}\left(m_{j} \cdot \tilde{b}_{j}\right) \geq 1+c\left|\sum_{\ell<j} \gamma_{\ell}\left(\tilde{b}_{\ell} \cdot m_{j}\right)\right| .
$$

Finally let $H=\sum_{j} \gamma_{j} \tilde{b}_{j}$. It is claimed, for all $j \geq 3$ that for any $m \in \mathcal{N}_{\varsigma}\left(m_{j}\right)$ the effect of $(H \cdot m)$ is pretty much of the order unity. Indeed, writing $m=m_{j}+\delta m$,

$$
(H \cdot m)=(H \cdot \delta m)+\gamma_{j}+\sum_{\ell<j} \gamma_{\ell}\left(\tilde{b}_{\ell} \cdot m_{j}\right),
$$

where terms of the form $\left(m_{j} \cdot \tilde{b}_{\ell}\right)$ with $\ell>j$ are absent due to orthogonality. Thus, it is clear, we now have $\left(H \cdot m_{j}\right) \geq 1-\varsigma\|H\|_{2}$.

Now, for $\lambda \in[-1,+1]$, consider the field $\tilde{b}_{1,2}(\lambda)=\lambda\left(\tilde{b}_{1}-\gamma_{2} \tilde{b}_{2}\right)$, where $\gamma_{2}$ is defined along the lines of the above $\gamma$ 's:

$$
\gamma_{2}=\gamma_{2}\left(\tilde{b}_{2} \cdot m_{2}\right)=1+\left|\left(\tilde{b}_{1} \cdot m_{2}\right)\right| \text {. }
$$

Obviously if $\lambda=1$, then $\left(\tilde{b}_{1,2}(1) \cdot m_{1}\right)=1$ and, as is seen, $\left(\tilde{b}_{1,2}(1) \cdot m_{2}\right) \leq-1$, $\left(\tilde{b}_{1,2}(-1) \cdot m_{1}\right)=-1$ while $\left(\tilde{b}_{1,2}(1) \cdot m_{2}\right) \geq 1$. Now let $\varepsilon_{1}, \varepsilon_{2}>0$ with $\varepsilon_{1} \gg \varepsilon_{2}$ (with the $\varepsilon$ 's to be specified with a bit more precision below) and consider $b_{\lambda}^{1,2}=-\varepsilon_{1} H+\varepsilon_{2} \tilde{b}_{1,2}(\lambda)$. For $m \in \mathcal{N}_{\zeta}\left(m_{1}\right)$, using $m=m_{1}+\delta m_{1}$, we have

$$
\left(m \cdot b_{\lambda}^{1,2}\right)=\varepsilon_{2}\left(\tilde{b}_{1,2}(\lambda) \cdot m_{1}\right)+\varepsilon_{2}\left(\tilde{b}_{1,2}(\lambda) \cdot \delta m_{1}\right)-\varepsilon_{1}\left(H \cdot \delta m_{1}\right),
$$

and we see that, at least for $|\lambda|$ near one, the second term can be neglected relative to the first. Similarly, if we allow $\varsigma \varepsilon_{1}$ small compared with $\varepsilon_{2}$, the third term may be designated as "unimportant". Of course the same considerations apply if $m \in \mathcal{N}_{\varsigma}\left(m_{2}\right)$. Meanwhile, if $m$ is in $\mathcal{N}_{\varsigma}\left(m_{j}\right)$ with $j \geq 3$, then

$$
\left(m \cdot b_{\lambda}^{1,2}\right)=-\varepsilon_{1}[(H \cdot m)]+\varepsilon_{2}\left(\tilde{b}_{1,2}(\lambda) \cdot m\right),
$$

so the first term is a negative number of order unity times $\varepsilon_{1}$ and, relative to this, the second term may be neglected due to the stipulation concerning the relative sizes of the 
$\varepsilon$ 's. Thus it is clear that the magnitude of the terms emerging from $\mathcal{N}_{\varsigma}\left(m_{3}\right), \ldots \mathcal{N}_{\varsigma}\left(m_{k}\right)$ are always much larger than those from magnetizations inside $\mathcal{N}_{\varsigma}\left(m_{1}\right)$ and $\mathcal{N}_{\varsigma}\left(m_{2}\right)$ and, according to the sign of the interaction, these $k-2$ regions are ruled out as candidates for the minimizer of the free energy function. Thus the minimum occurs in $\mathcal{N}_{\zeta}\left(m_{1}\right) \cup \mathcal{N}_{\zeta}\left(m_{2}\right)$ and it must be the case that the minimizer switches locations for some $\lambda \in(-1,+1)$.

Thus for the interaction given by $-\beta \mathcal{H}+\sum_{i}\left(b_{\lambda}^{1,2} \cdot \mathbf{s}_{i}\right)$ it is clear that the associated mean-field theory has a Generic First Order Scenario - albeit field driven; see Remark 2 following Definition 2.1. Indeed, for fixed small $\varsigma$, and $\left(\varepsilon_{1}, \varepsilon_{2}\right)$ chosen accordingly, it follows from continuity (cf. Theorem 5.11) there is at least one $\lambda_{\mathrm{MF}}=\lambda_{\mathrm{MF}}\left(\varepsilon_{1}, \varepsilon_{2}\right)$ with $\lambda_{\mathrm{MF}} \in(-1,+1)$, where the minimum in $\mathcal{N}_{\varsigma}\left(m_{1}\right)$ coincides with the minimum in $\mathcal{N}_{\zeta}\left(m_{2}\right)$. To define $\mathbb{M}_{I}$ and $\mathbb{M}_{I I}$ we restrict to the subsets of $\mathcal{N}_{\zeta}\left(m_{1}\right)$ and $\mathcal{N}_{\zeta}\left(m_{2}\right)$ such that hypothesis (iic) is satisfied and then (iia), (iib) and (iid) are easily satisfied. The remains of this proof now follow from the Main Theorem.

\subsection{Proofs for specific systems. Let us start with the standard discrete symmetry mag- netic transitions:}

Proof of Theorems 4.1 and 4.2. These systems (as well as a host of others) may be treated together since, in fact, the principal results pertaining to the nature, location etc. of the first order transition are just an application of Theorem (3.2). The secondary result, namely that the high/low temperature states "disappear" on the appropriate side of $\beta_{t}$ is also, in fact a fairly general feature of these sorts of systems but not really worth abstractifying.

Let us start with some basic facts about the mean-field theory which are well known and/or readily derived (and anyway proved in [2], Sect. 4.2 and Sect. 4.3 ). Foremost, for $q \geq 3$ and $r \geq 4$ there is indeed a first order transition in the mean-field theory; the temperature parameter will in all cases be denoted by $\beta_{t}^{\mathrm{MF}}$. In both cases the degenerate minima consist of singleton positive magnetization states which are proportional to the values that the spins themselves take as well as a state of zero magnetization. These obviously enact the symmetries of the relevant groups and, needless to say are convex sets. The energy gap is manifest and in addition, it is worth noting that the aforementioned $\beta_{t}^{\mathrm{MF}}$ is the only point of degeneracy between states of differing energy. Thus we apply Theorem 3.2.

As for the "disappearance of states", this follows from elementary considerations. In particular, in the real system, the energy is a monotone function (and so a.e. well defined). Thus, for $\beta<\beta_{t}$ there cannot be any states with large magnetization - since that would imply the existence of a substantial energy - and similarly when $\beta>\beta_{t}$ there cannot be states with small magnetization.

On to the asymmetric situation: To prove the content of Theorem 4.3 it is, by and large, sufficient to establish a triple point in the context of the mean-field theory. The claim, for the mean-field theory, is best summarized in Fig. 2 below and will be proved as a separate lemma.

Lemma 5.10. Consider the mean-field theory associated with the Hamiltonian 4.1 which leads to the mean-field free energy function

$$
\begin{aligned}
\Phi_{\mathbf{J}}\left(n_{a}, n_{b}, n_{c}\right)= & -\frac{1}{2} J_{a} n_{a}^{2}-\frac{1}{2} J_{b} n_{b}^{2}-\frac{1}{2} J_{c} n_{c}^{2}-K_{b c} n_{b} n_{c}+K_{a b} n_{a} n_{b} \\
& +n_{a} \log n_{a}+n_{b} \log n_{b}+n_{c} \log n_{c},
\end{aligned}
$$




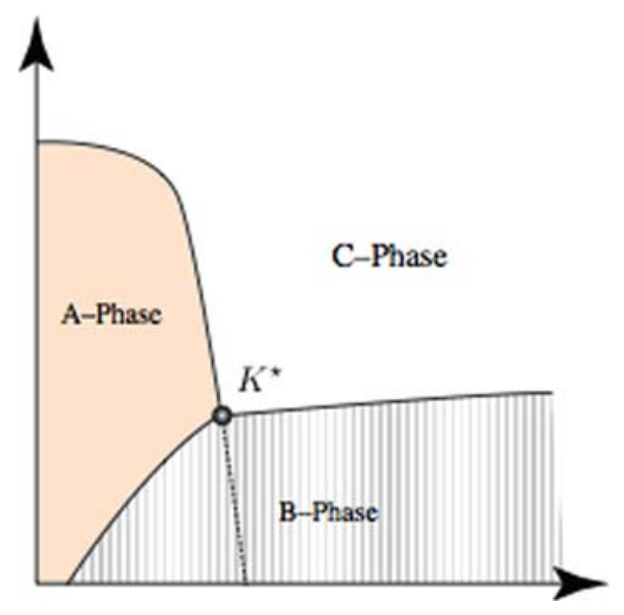

Fig. 2. Phase diagram for an asymmetric 3-state model

where $n_{a}+n_{b}+n_{c}=1$ and $\mathbf{J}$ stands for all the couplings. Using the notations $D_{a}$, $D_{b}$ and $J$ as described, the following holds for all $J>\tilde{J}$ where $\tilde{J}$ is large (but not unreasonably so):

1. For all $D_{a}$ and $D_{b}>0$ and for all $K_{a b}$ and $K_{b c} \geq 0$ with the $K$ 's small compared to $J$ there are three local minima, at least one of which is the global minimum, that are characterized by an abundance of the species $a, b$ and $c$ respectively. These minima will correspondingly be denoted by $A, B$ and $C$ and when they actually minimize they represent the phases. Any other local minima of $\Phi_{\mathbf{J}}$ are substantially higher.

2. For fixed $D_{a}$ and $D_{b}$ sufficiently small compared to $J$ and $\left(D_{a}-D_{b}\right)$ small compared to $D_{a}$, there is a finite $\tilde{K}$ such that for $\tilde{K}>K_{a b}$, and $K_{b c}$ sufficiently small, the A-phase is the minimizer. By contrast, for small values of $K_{a b}$ the B-phase will be prevalent once $K_{b c}$ is sufficiently large.

3. With $D_{a}$ and $D_{b}$ as above, for all $K_{a b}<\tilde{K}$, there is a transitional point at some value of $K_{b c}$, where the $A$ and $C$ minima are degenerate. Pertinently, this holds even in the B phase. Furthermore, these points form a "transitional curve" which cuts through the B-phase. The terminal point of this curve in the B-phase is the point $K^{\star}$.

Proof of Lemma 5.10. Let us start with the situation $D_{a}=\cdots=K_{b c}=0$ - i.e. the $q=3$-state Potts model - with $J$ in excess of some $\tilde{J}$ to be described later. The claim is that there are three minimizers, identical under permutation, with one large and two small populations. While this is of course well known, the forthcoming analysis will demonstrate that these solutions are stable and persistent. Moreover, other local minima (if any) will have substantially higher free energy and/or represent unphysical states.

The starting point is, of course, the mean-field equation:

$$
n_{a} e^{-J n_{a}}=n_{b} e^{-J n_{b}}=n_{c} e^{-J n_{c}}=\lambda,
$$

where $\lambda$ is a Lagrange multiplier adjusted so that $n_{a}+n_{b}+n_{c}=1$. A look at the function $x e^{-J x}$ clearly indicates that for $\lambda<(J e)^{-1}$, there are two solutions to $x(\lambda) e^{x(\lambda)}=\lambda$ which, for obvious reasons will be denoted by $s(\lambda)$ and $B(\lambda)$. Note that $s(\lambda)$ is strictly 
increasing on $\left[0,(J e)^{-1}\right]$ while $B(\lambda)$ is strictly decreasing. The objective, at the level of the $q$-state Potts model, is to find the value of $\lambda$ such that $(q-1) s+B=1$. That such a value exists is obvious; as $\lambda \rightarrow 0, B \rightarrow \infty$ while for the maximum value, $\lambda=(J e)^{-1}$, $B=s=1 / J$ - can be assumed to be way too small. So, by "bringing up" the value of $\lambda$, we certainly arrive at a first solution; the claim is that for $J$ in excess of some value $\tilde{J}$ this is (far and away) the only possibility.

Direct computation yields

$$
\dot{B}+(q-1) \dot{s}=\frac{(q-1) s}{1-J s}-\frac{B}{J B-1},
$$

where the overdot denotes differentiation with respect to $\log \lambda$. For $q=2$ it may be directly verified that this is negative but not for $q \geq 3$. However, this $i s$ negative till $q J s B \geq B+(q-1) s$. Now the latter necessarily implies $q J s B>B$, i.e. $J s$ is already of order unity. But then so is $J B$; indeed, under the previously mentioned condition,

$$
J \lambda=J s e^{-J s} \geq \frac{1}{q} e^{-\frac{1}{q}},
$$

thence

$$
J \lambda=J B e^{-J B} \leq \frac{1}{q} e^{-\frac{1}{q}}
$$

To summarize: For $J$ in excess of some $\tilde{J}$ - not terribly large - there is no hope of a second solution to $B+(q-1) s=1$ because by the time the derivative of $B+(q-1) s$ gets around to being positive, all $B$ 's and $s$ 's are "hopelessly small", namely of order $J^{-1}$. The only other possibility for minima are two (or more) $B$-type solutions. However, under these circumstances, it has been shown that the free energy is substantially lowered if, keeping all other $n$ 's fixed, two bigs are exchanged for a small and a (bigger) big; cf. the proof of Lemma 4.4, especially item (i), in [2].

It is clear that the above analysis all goes through with different diagonal couplings, e.g. $J_{a}>J_{b}>J_{c}$. Let us proceed with the full problem. While we will not use that $D_{a}$ is small compared with $J$ until later, it is conceptually easier to proceed in this vein. The full equations now read

$$
n_{a} e^{-J n_{a}} e^{+K_{a b} n_{b}}=n_{b} e^{-J n_{b}} e^{+K_{a b} n_{a}} e^{-K_{b c} n_{c}}=n_{c} e^{-J n_{c}} e^{-K_{b c} n_{b}}=\lambda
$$

we are seeking solutions of the form "two small one big”. First off, let us note that there are some restrictions on $\lambda$. For example, the third equation certainly requires $\lambda<(\mathrm{Je})^{-1}$ and further, for $\lambda$ comparable to this number, it is easily seen that if there were a solution, it would have (for $\left.K_{a b}, K_{b c} \ll J\right) n_{a}+n_{b}+n_{c}$ of the order $J^{-1}$. So we shall restrict attention to, say, $\lambda$ less than $\lambda_{0}=\kappa(J e)^{-1}$ with some suitably chosen $\kappa$ of order unity but less than one and proceed.

It is not hard to see that there is indeed a unique solution once the big item is specified. Suppose, for example, this is $n_{a}$. Let us write a facsimile of the first equation, namely $N_{a} e^{-J_{a} N_{a}} e^{K_{a b} n_{b}}=\lambda$, which defines a function $N_{a}\left(n_{b}\right)$. It so happens that this is defined on all of $[0, \infty)$ but not so for $N_{c}\left(n_{b}\right)$ given from the third equation: $N_{c} e^{-J_{c} N_{c}} e^{K_{b c} n_{b}}=\lambda$. However, for $\lambda<\lambda_{0}$, the quantity $n_{b}$ can safely climb up to the order of $J^{-1}$ which, as we shall see, is more than ample range. From the middle equation, we can now define a function

$$
\Lambda\left(n_{b}\right)=n_{b} e^{-J_{b} n_{b}} e^{+K_{a b} N_{a}\left(n_{b}\right)} e^{-K_{b c} N_{c}\left(n_{b}\right)},
$$


and we now wish to solve $\Lambda=\lambda$. Clearly $N_{b}(0)=0$ while, as $n_{b}$ tends to the order of $J^{-1}$, the right-hand side will exceed $\lambda_{0}$. (Ignoring the aid from the $e^{+K_{a b} N_{a}\left(n_{b}\right)}, N_{c}$ does not get any bigger than $J^{-1}$ so $\Lambda$ can certainly get almost all the way up to $(J e)^{-1}$.) Thus, for all $\lambda$ of interest there is a solution $n_{a}(\lambda), n_{b}(\lambda), n_{c}(\lambda)$. To see that it is unique (at least for the values of $\lambda$ that are of interest) we can simply take the derivative:

$$
\frac{d}{d n_{b}} \log \Lambda\left(n_{b}\right)=\frac{1}{n_{b}}-J_{b}+K_{a b} N_{a}^{\prime}-K_{b c} N_{c}^{\prime} .
$$

Now $N_{a}$ is increasing but so (unfortunately) is $N_{c}$. However, in order for $K_{b c} N_{c}^{\prime}=$ $K_{b c}^{2}\left(N_{c}^{-1}-J_{c}\right)^{-1}$ to be comparable with $n_{b}^{-1}-J_{b}$, it is obvious that $n_{b}^{-1}$ and $J_{b}$ must themselves be comparable which puts $\Lambda$ in well excess of $\kappa_{0}$. Notwithstanding, even when $n_{b} \approx J^{-1}, N_{c}\left(n_{b}\right)$ is still small compared with $J^{-1}$, and hence given the rest of the range of $n_{b}$ the negative portion of the derivative is not substantial enough to pull the function down below $\kappa \lambda_{0}$. Item 1 has essentially been proved: Having established, e.g. for $a$ dominance over $b$ and $c$ the existence of unambiguous $n_{a}(\lambda), n_{b}(\lambda)$ and $n_{c}(\lambda)$, an argument similar to the $K_{a b}=K_{b c}=0$ case shows the existence of a unique $\lambda$ such that $n_{a}+n_{b}+n_{c}=1$. Similarly for the other orderings. Thus, in the region of parameters described, we now have our three well defined "free energies", $\Phi_{\mathrm{A}, \mathbf{J}}, \Phi_{\mathrm{B}, \mathbf{J}}$ and $\Phi_{C, J}$ associated with these three (well separated) local minima. At least one of these functions will represent the actual $F_{\mathrm{MF}}$ and all of them are substantially lower than any other value of $\Phi_{\mathbf{J}}$ outside the vicinity of the minima.

Items 2 and 3 are actually not so difficult in light of what has already been established. Indeed, it is observed that the derivatives of the various free energy functions with respect to the couplings admit simple expressions due to the fact that they are already functions evaluated at local minima. For example let us examine $\Phi_{\mathrm{A}, \mathbf{J}}$ expressed in the form of Eq. (5.10) with $n_{c}$ formally eliminated in terms of $n_{a}$ and $n_{b}$. Then

$$
\frac{\partial \Phi_{\mathrm{A}, \mathbf{J}}}{\partial K_{a b}}=n_{a}(\mathrm{~A}) n_{b}(\mathrm{~A})+\left[\frac{\partial \Phi_{\mathbf{J}}}{\partial n_{a}}\right]_{\mathrm{A}} \frac{\partial n_{a}}{\partial K_{a b}}+\left[\frac{\partial \Phi_{\mathbf{J}}}{\partial n_{b}}\right]_{\mathrm{A}} \frac{\partial n_{b}}{\partial K_{a b}},
$$

where the subscripts and arguments of A for various quantities emphasize that the associated functions should be evaluated at the portions of $n_{a}$ and $n_{b}$ (and $n_{c}=1-n_{a}-n_{b}$ ) which produce the A-state. However here the relevant partial derivatives vanish because we have a local minimum. Hence $\partial \Phi_{\mathrm{A}, \mathbf{J}} / \partial K_{a b}$ is simply $n_{a} n_{b}-$ as evaluated in the $A$-state. These derivative arguments will greatly facilitate the proof of all that remains.

More pertinent than the above equation is that

$$
\frac{\partial \Phi_{\mathrm{C}, \mathbf{J}}}{\partial K_{b c}}=-n_{b}(C) n_{c}(C)
$$

with a formally identical expression for the same derivatives of $\Phi_{\mathrm{B}, \mathbf{J}}$ and $\Phi_{\mathrm{A}, \mathbf{J}}$ but with the right-hand side given by the product of the $n$ 's evaluated in the appropriate states. It is noted that for all $K_{a b}, K_{b c}$ of relevance, $n_{b}(A) n_{c}(A) \ll n_{b}(B) n_{c}(B), n_{b}(C) n_{c}(C)$. For $K_{b c}=0$, it is clear that until $K_{a b}$ has become substantial the A-phase is dominant. ${ }^{1}$ For $K_{a b}$ small, it is clear that once $K_{b c}$ gets large enough, the $\Phi_{\mathrm{A}}$ and $\Phi_{\mathrm{B}}$ minima will exchange. Thus, in the vicinity of the origin of the $K$-space quadrant, the vertical

\footnotetext{
1 In the absence of additional analysis/analytics, the current argument may represent an unmentioned and somewhat non-trivial requirement: For large $J$, we have $s(J) \approx e^{-J}$ and since the perturbations must always couple to a subdominant species in order for the $K$ 's to have impact without themselves becoming unreasonably large it is actually required that $D_{a} e^{J}$ be somewhat small.
} 
axis is enveloped by the A-phase while most of the horizontal axis (and its immediate neighborhood) is dominated by the B-phase. This is item 2 .

As for item 3, let us start on the vertical axis at a point in the A-phase. Now, we simply compare the derivative in Eq. (5.48) with the counterpart derivative of $\Phi_{\mathrm{A}}$ : Under the restrictions in the statement of this theorem, the former is always larger in magnitude than the latter. Thus, if $D_{a}$ has been arranged to be suitably small, it is inevitable that $\Phi_{C}$ will "catch up" and once it has done so, it will always "stay ahead". This defines a transitional point which, it is reiterated, may or may not happen within the region of the B-phase. The fact that these points form a curve follows from an elementary argument using (compactness and) the implicit function theorem.

Proof of Theorem 4.3. Taking $\Omega$ to be the standard positive basis vectors in $\mathbb{R}^{3}$, as long as the $K$ 's are not comparable to $J$, (which is anyway required later) the interaction describes a positive definite inner product. Under the conditions of Lemma 5.10 let us assume, for fixed $J$ 's, that the $K$ 's are adjusted so that the mean-field theory is at the point $K^{\star}$. The occupation vectors corresponding to the $A, B$ and $C$ phases are manifestly seen to be linearly independent - regarded as vectors in $\mathbb{R}^{3}$ - and are well separated in $(\operatorname{Conv}(\Omega))$ since each of the vectors has a dominant component. All the conditions of Theorem 3.3 are satisfied; the result follows.

Proof of Theorem 4.4. Of course much of the statement of this theorem amounts to a statement about the mean-field theory and this system is well characterized. A brief run through will be provided for completeness. If $m \in \Omega_{n}$ - the unit sphere in $n$-dimensions - obvious symmetry considerations reduce all considerations to scalar problems. Thus, e.g. the function $\mathrm{m}_{n}(h)$ is given by Eq. (4.2) and once computations are performed, all quantities can be promoted to vectors. Using $h$ in favor of $m$, (see Proposition 5.12 in the Appendix subsection) the expression for the free energy may be written

$$
\Phi_{\beta}=-\frac{1}{2} \beta m^{2}-\log G(h)+m h,
$$

where all terms involving $m$ are now understood to mean $\mathrm{m}_{n}(h)$. Then $\Phi_{\beta}^{\prime}=[h-\beta m] m^{\prime}$ and noting that $m^{\prime}$ is strictly positive, for all intents and purposes, its presence can be ignored. We are, of course, running through a derivation of the mean-field equation and so far everything is, more or less, general. The specifics for this problem is that $\mathrm{m}_{n}(h)$ is a strictly convex function $[13,28]$. Now it turns out that $\lim _{h \rightarrow 0} \frac{\beta_{c} \mathrm{~m}_{n}(h)}{h}=1$ with $\beta_{c}=n$. Strict concavity gives us that for positive $h, \beta_{c} \mathrm{~m}_{n}(h) \leq h$ so that if $\beta<\beta_{c}$, the free energy is raised by making $h$ positive, i.e. $m(\beta)=0$. Conversely, if $\beta>\beta_{c}$, raising $h$ away from zero will lower the free energy which continues until the mean-field equation is satisfied. The solution is demonstrably unique by the concavity property and obviously a minimum. The comparison with the actual spin-systems is a direct consequence of Lemma 5.1 and Corollary 5.3, the claims concerning the energy follow from Proposition 5.7; due to the continuous nature of the transition, the Regular Energy Hypothesis is obvious with $r=1$ and, finally, the statement concerning the free energy is exactly Corollary 5.3 to Lemma 5.1 .

Proof of Theorem 4.5. Practically all of what is needed is contained in the second (substantial) half of Theorem 3.2: The appropriate $\Xi_{\eta}$ sets are neighborhoods of the origin and the orbit of $\lambda_{t}^{\mathrm{MF}} \operatorname{diag}\left[1,-\frac{1}{\mathrm{n}-1}\right]$ under the action of the full $O(n)$ group. These sets are obviously separated in magnetization and energy. Of course the origin is a singleton - convex - so the "magnetic" portions of Theorem 3.2 actually apply which is the 
entirety of the claim concerning the high temperature phase. Of course, the low temperature portion of $\mathcal{M}_{\beta_{t}^{\mathrm{MF}}}$ is not a convex set. Thus while (at least in the matrix version) the block magnetizations on block scale $\ell_{0}$ appear like the mean-field minimizers, there is no reason to expect this sort of coherence on larger scales. A global cooperative effect requires additional ingredients which are present in $d>3$ but most definitively absent in $d=2$.

Proof of Theorem 4.6. The mean-field bound for these systems is actually standard fare: For the Ising system, it follows from an adaptation of a general result to this effect by Sokal [33]. For $n$-component spins, with any non-negative $J_{i, j}$ it was shown in [32] that the following inequality holds for $n \geq 2$ :

$$
\left\langle\mathbf{s}_{i}^{(1)}\right\rangle_{\Lambda, h} \leq \frac{\beta}{n} \sum_{j} J_{i, j}\left\langle\mathbf{s}_{j}^{(1)}\right\rangle_{\Lambda, h}+h,
$$

where the superscripts here denote the first component and $\langle-\rangle_{\Lambda, h}$ denotes the thermal average in system $\Lambda$ (with certain boundary conditions) at external field $h$ pointing in the direction of the first component. In point of fact, this also holds for $n=1-$ at least for $h=0-$ where it is the Simon inequality [31] in slightly disguised form. Since it is well known for $n=1$ and known [8] for $n=2$ how to provide the appropriate boundary conditions for producing the spontaneous magnetization, we might as well take the inequality as it stands with $n=1$ or $2, h=0$ and $\Lambda \rightarrow \infty$ replacing thermal averages of spin components by spontaneous magnetizations. In the present context, this reads

$$
m_{\alpha} \leq \frac{\beta}{n} Q^{\alpha, \gamma} m_{\gamma}
$$

where $m_{\alpha}$ (with $m_{\alpha} \geq 0$ ) denotes the spontaneous magnetization in the $\alpha^{\text {th }}$ layer. The result now follows pretty easily if we multiply by $m_{\alpha}$ and sum over $\alpha$ (cf. [10] for a more detailed derivation along these lines). All the rest of the claims now follow from previous theorems. For $\mu$ small, the block magnetizations are (uniformly on compact intervals of temperature) close to a solution of the mean-field equation by Proposition 5.1 and its corollary. Free energetics and energetics follow from Corollary 5.3 and Proposition 5.7 (where we may use $r=1$ because the transition is continuous) and observe that the Regular Energy Hypothesis satisfied.

5.5. Appendix: Continuity properties of $\Phi_{\beta}(m)$. Here are some properties of the free energy function that have been alluded to, or explicitly used in the text. The starting point will be to trim away the inessential portions of $\mathbb{E}_{\Omega}$ and even $\Omega$, which will later save us the trouble of numerous provisos. Let $\mathbb{D}_{\Omega}$ denote the set

$$
\mathbb{D}_{\Omega}=\left\{h \in \mathbb{E}_{\Omega} \mid(\mathbf{s} \cdot h)=\text { constant w.p. } 1\right\} .
$$

Obviously $\mathbb{D}_{\Omega}$ is a subspace of $\mathbb{E}_{\Omega}$ and it is seen, after a moments thought, that the non-trivial vectors in $\mathbb{D}_{\Omega}$ are precisely the ones that are of no interest to the problem at hand. The price of keeping $\mathbb{D}_{\Omega}$ is that relative topologies must be employed and many statements must be made modulo vectors in $\mathbb{D}_{\Omega}$. Thus, without loss of generality, we restrict attention to the essential subspace and, without much apology, continue with the notations $\Omega, \mathbb{E}_{\Omega}$, etc. But, for future reference it is now noted that

$$
(\mathbf{s} \cdot h)=0 \text { constant w.p. } 1 \Rightarrow h=0 \text {. }
$$

The principal result of this section is the continuity of $\Phi_{\beta}$ : 
Theorem 5.11. Let $\Omega, \mathbb{E}_{\Omega}$ and $\alpha_{0}$ be as described with the stipulation in Eq. (5.53) and let $\mathscr{C}_{\Omega}$ denote the set where the entropy is not $-\infty$. Then the free energy function,

$$
\Phi_{\beta}(m)=-\frac{1}{2} \beta m^{2}-(b \cdot m)-S(m),
$$

is continuous on $\operatorname{Int}\left(\mathscr{C}_{\Omega}\right)$.

As a starting point, for $m \in \mathbb{E}_{\Omega}$, let us define the entropy functional

$$
\Psi_{m}=\frac{e^{(m \cdot h)}}{\int_{\Omega} e^{(\mathbf{s} \cdot h)} d \alpha_{0}}=e^{(m \cdot h)-G(h)} .
$$

The object is to maximize $\Psi_{m}$. Obviously $\mathscr{C}_{\Omega}$ is the set where $\Psi_{m}$ is bounded; it is not hard to see that $\mathscr{C}_{\Omega} \subset \operatorname{Conv}(\Omega)$. For $h \in \mathbb{E}_{\Omega}$ let us use the notation $\langle-\rangle_{h}$ for expectation in the tilted measure and define $\mathbf{m}(h)\left(=\langle\mathbf{s}\rangle_{h}\right)$ to be the average magnetization in this measure. In [2] it was proved (Lemma 3.1) that if $m \in \operatorname{Int}\left(\mathscr{C}_{\Omega}\right)$, then $\exists h \in \mathbb{E}_{\Omega}$ such that $\mathbf{m}(h)=m$. Here let us prove that this $h$ is unique.

Proposition 5.12. Let $\Omega, \mathbb{E}_{\Omega}, \alpha_{0}$ and $\mathscr{C}_{\Omega}$ be as described, with the stipulation in Eq. (5.53). Let $m \in \mathbb{E}_{\Omega}$ satisfy $\mathbf{m}(h)=m$ for some $h \in \mathbb{E}_{\Omega}$. Then, in fact, $m \in \mathscr{C}_{\Omega}$ and the $h$ is unique.

Proof. The fact that $h \in \mathscr{C}_{\Omega}$ was proved in [2] Lemma 3.1 - but also follows from the argument below which, in fact, is almost exactly the proof of Theorem 2.4 in [9]. In any case, we have, from the above-mentioned lemma in [2] that $h$ maximizes $\Psi_{m}$. Suppose that $\tilde{h}$ also satisfies $m=\mathbf{m}(\tilde{h})$. Then

$$
\begin{aligned}
\Psi_{m}(h) & =\frac{e^{(m \cdot \tilde{h})} e^{(m \cdot[h-\tilde{h}])} e^{G(\tilde{h})}}{e^{G(h)} e^{G(\tilde{h})}}=\Psi_{m}(\tilde{h}) \frac{e^{G(\tilde{h})}}{\int_{\Omega} e^{(\mathbf{s} \cdot \tilde{h})} e^{(\mathbf{s} \cdot[h-\tilde{h}])} d \alpha_{0}} e^{(m \cdot[h-\tilde{h}])} \\
& =\Psi_{m}(\tilde{h}) \frac{e^{(m \cdot[h-\tilde{h}])}}{\left\langle e^{\mathbf{s} \cdot[h-\tilde{h}]}\right\rangle_{\tilde{h}}} \leq \Psi_{m}(\tilde{h}) e^{\left(\langle\mathbf{s}\rangle_{\tilde{h}} \cdot[h-\tilde{h}]\right)} e^{(m \cdot[h-\tilde{h}])}=\Psi_{m}(\tilde{h}),
\end{aligned}
$$

where the inequality is Jensen's. Evidently $\tilde{h}$ also maximizes the functional. Moreover, since the Jensen inequality has saturated, $(\mathbf{s} \cdot[h-\tilde{h}])$ is a.s. a constant according to the tilted measure and hence according to $\alpha_{0}$. Evidently $h=\tilde{h} \alpha_{0}-$ a.s.

The above proposition allows the definition of an inverse function $\mathbf{h}(m)$ defined, at least, on $\operatorname{Ran}(\mathbf{m})$. The next result shows that $\mathbf{h}$ is continuous:

Proposition 5.13. Let $\Omega, \mathbb{E}_{\Omega}, \alpha_{0}$ and $\mathscr{C}_{\Omega}$ be as described, with the stipulation in Eq. (5.53). Then $\operatorname{Ran}(\mathbf{m})=\operatorname{Int}\left(\mathscr{C}_{\Omega}\right)$ wherein the inverse map $\mathbf{h}$ is continuous.

Proof. This follows from standard convexity arguments. For example, if $m \in \operatorname{Ran}(\mathbf{m})$ and it is assumed, with no loss of generality (although, perhaps, some elegance) that by linear transformation the problem has been reduced to $n$-dimensional Euclidean with standard inner product then the derivative is, explicitly,

$$
\frac{\partial m_{a}}{\partial h_{b}}=\left\langle s_{a} s_{b}\right\rangle_{h}-\left\langle s_{a}\right\rangle_{h}\left\langle s_{b}\right\rangle_{h},
$$


where $h=\mathbf{h}(m)$. However, the object in Eq. (5.57) is exactly the covariance matrix of the "array" $\mathbf{s}$ in the tilted measure. In general this is positive semi-definite but due to the stipulation in Eq. (5.53), it is positive definite. Hence the inverse function is itself differentiable and, moreover, any point in a sufficiently small neighborhood of $m$ can be reached by $\mathbf{h}$. Now by [2] Lemma 3.1 we have that $\operatorname{Int}\left(\mathscr{C}_{\Omega}\right) \subset \operatorname{Ran}(\mathbf{m}) \subset \mathscr{C}_{\Omega}$ but the latter argument tells us that $\operatorname{Int}\left(\mathscr{C}_{\Omega}\right) \supset \operatorname{Ran}(\mathbf{m})$.

As an obvious corollary:

Proof of Theorem 5.11. Clearly, it is only necessary to establish continuity of $S(m)$. However, we may now express

$$
S(m)=G(\mathbf{h}(m))-(m \cdot \mathbf{h}(m)),
$$

and the continuity of both portions follows from the continuity of $\mathbf{h}(m)$.

Acknowledgements. This research was supported by the NSF under the grant DMS-0306167.

Open Access This article is distributed under the terms of the Creative Commons Attribution Noncommercial License which permits any noncommercial use, distribution, and reproduction in any medium, provided the original author(s) and source are credited.

\section{References}

1. Biskup, M.: Reflection Positivity and Phase Transitions in Lattice Spin Models. Lecture notes from Prague Summer School on Mathematical Statistical Mechanics, 2006

2. Biskup, M., Chayes, L.: Rigorous analysis of discontinuous phase transitions via mean-field bounds. Commun. Math. Phys. 238(1), 53-93 (2003)

3. Biskup, M., Chayes, L., Crawford, N.: Mean-field driven first-order phase transitions in systems with long-range interactions. J. Stat. Phys. 119(6), 1139-1193 (2006)

4. Biskup, M., Chayes, L., Nussinov, Z.: Orbital ordering in transition-metal compounds: I. the 120-degree model. Commun. Math. Phys. 255, 253-292 (2005)

5. Biskup, M., Kotecký, R.: Forbidden gap argument for phase transitions proved by means of chessboard estimates. Commun. Math. Phys. 264(3), 631-656 (2006)

6. Butta, P., Picco, P.: Large-deviation principle for one-dimensional vector spin models with Kac potentials. J. Stat. Phys. 29(1/2), 101-150 (1998)

7. Cassandro, M., Orlandi, E., Presutti, E.: Interfaces and typical Gibbs configurations for one-dimensional Kac potentials. Probab. Theory Relat. Field 96, 57-96 (1993)

8. Chayes, L.: Discontinuity of the spin-wave stiffness in the two-dimensional XY model. Commun. Math. Phys. 197, 623-640 (1998)

9. Chayes, J.T., Chayes, L., Lieb, E.H.: The inverse problem in classical statistical mechanics. Commun. Math. Phys. 93, 57-121 (1984)

10. Zandi, R., Shackell, A., Rudnick, J., Kardar, M., Chayes, L.P.: Thinning of superfluid films below the critical point. Phys. Rev. E 76, 030601 (R) (2007)

11. Dembo, A., Zeitouni, O.: Large Deviations Techniques and Applications. New York: Springer-Verlag Inc., 1998

12. Dobrushin, R.L., Shlosman, S.: Absence of breakdown of continuous symmetry in two-dimensional models of statistical physics. Commun. Math. Phys. 42, 31-40 (1975)

13. Ellis, R.S., Monroe, J.L., Newman, C.M.: The GHS and other correlation inequalities for a class of even ferromagnets. Commun. Math. Phys. 46(2), 167-182 (1976)

14. Frohlich, J., Spencer, T.: The Kosterlitz-Thouless phase transition in two-dimensional abelian spin-systems and the coulomb gas. Commun. Math. Phys. 81, 527-602 (1981)

15. Garcia, R., Chan, M.H.W.: Critical fluctuation-induced thinning of ${ }^{4} \mathrm{He}$ films near the superfluid transition. Phys. Rev. Lett. 83(6), 1187-1190 (1998)

16. Ganshin, A., Scheidemantel, S., Garcia, R., Chan, M.H.W.: Critical casimir force in ${ }^{4} H e$ films: confirmation of finite-size scaling. Phys. Rev. Lett. 97, 075301 (2006)

17. Gambassi, A., Dietrich, S.: Critical dynamics in thin films. J. Stat. Phys. 123(5), 929-1005 (2006) 
18. Gobron, T., Merola, I.: First-order phase transition in potts models with finite-range interactions. J. Stat. Phys. 126, 507-583 (2007)

19. Hohenberg, P.C.: Existence of long-range order in one and two dimensions. Phys. Rev. 158, 383-386 (1967)

20. Kennedy, T., Lieb, E.H., Shastry, B.S.: Existence of Néel order in some spin 1/2 Heisenberg antiferromagnets. J. Stat. Phys. 53, 1019-1030 (1988)

21. Kesten, H., Schonmann, R.: Behavior in large dimensions of the potts and Heisenberg models. Rev. Math. Phys. 1, 147-182 (1990)

22. Kosterlitz, J.M., Thouless, D.J.: Ordering, metastability and phase transitions in two-dimensional systems. J. Phys. C 6, 1181-1203 (1973)

23. Kotecký, R., Shlosman, S.B.: First-order phase transitions in large entropy lattice models. Commun. Math. Phys. 83, 493-515 (1982)

24. Lee, D.H., Caflisch, R.G., Joannopoulos, J.D.: Antiferromagnetic classical XY model: A mean-field analysis. Phys. Rev. B 29(5), 2680-2684 (1984)

25. Mayer, D.H.: The Ruelle-Araki Transfer Operator in Classical Statistical Mechanics. Lecture Notes in Physics, Vol. 123, Berlin: Springer Verlag, 1980

26. Mermin, D., Wagner, H.: Absence of ferromagnetism or antiferromagnetism in one-or two-dimensional isotropic Heisenberg models. Phys. Rev. Lett. 17, 1133-1136 (1966)

27. Nakanishi, H., Fisher, M.E.: Critical Point Shifts in Films. J. Chem. Phys. 78(6), Part I, 15 March 1983

28. Pearce, P.W.: Mean-field bounds on the magnetization for ferromagnetic spin models. J. Stat. Phys. 25(2), 309-320 (1981)

29. Pearce, P.W., Thompson, C.J.: The anisotropic Heisenberg model in the long-range interaction limit. Commun. Math. Phys. 41(2), 191-201 (1975)

30. Shlosman, S.B.: The method of reflection positivity in the mathematical theory of first-order phase transitions. Russ. Math. Surv. 41(3), 83-134 (1986)

31. Simon, B.: Correlation inequalities and the decay of correlations in ferromagnets. Commun. Math. Phys. 77(2), 111-126 (1980)

32. Simon, B.: The Statistical Mechanics of Lattice Gases. Vol. I., Princeton Series in Physics, Princeton, NJ: Princeton University Press, 1993

33. Sokal, A.D.: Mean-field bounds and correlation inequalities. J. Stat. Phys. 28(3), 431-439 (1982)

34. Wu, F.Y.: The potts model. Rev. Mod. Phys. 54, 235-268 (1982)

Communicated by H. Spohn 